\title{
Audio Features for Music Emotion Recognition: a Survey
}

\author{
Renato Panda, Ricardo Malheiro and Rui Pedro Paiva
}

\begin{abstract}
The design of meaningful audio features is a key need to advance the state-of-the-art in Music Emotion Recognition (MER). This work presents a survey on the existing emotionally-relevant computational audio features, supported by the music psychology literature on the relations between eight musical dimensions (melody, harmony, rhythm, dynamics, tone color, expressivity, texture and form) and specific emotions. Based on this review, current gaps and needs are identified and strategies for future research on feature engineering for MER are proposed, namely ideas for computational audio features that capture elements of musical form, texture and expressivity that should be further researched. Finally, although the focus of this article is on classical feature engineering methodologies (based on handcrafted features), perspectives on deep learning-based approaches are discussed.
\end{abstract}

Index Terms-affective computing, music emotion recognition, audio feature design, music information retrieval

\section{INTRODUCTION}

$\mathrm{M}$ usic Emotion Recognition (MER) is attracting increasing interest from the Music Information Retrieval (MIR) research community. In fact, as pointed out by David Huron nearly 20 years ago, "music's preeminent functions are social and psychological", and so "the most useful retrieval indexes are those that facilitate searching in conformity with such social and psychological functions. Typically, such indexes will focus on stylistic, mood, and similarity information" [1].

There is already a significant corpus of research on different aspects of MER, e.g., classification using symbolic files [2], single-label classification using raw audio excerpts [3-5], multi-label classification [6-7], dimensional approaches using regression $[8,9]$, music emotion variation detection [10, 11], lyrics-based MER [9], bimodal/multimodal approaches [2, 4], following either classical handcrafted feature design and machine learning [5] or deep learning [10] approaches, with specific MER datasets, e.g., $[5,8,11]$. Nevertheless, several limitations and problems still need to be addressed [5].

Most recent studies have devoted their attention to the MER problems above, datasets and improved machine learning techniques, while applying already existing audio features developed in other contexts, such as speech recognition or music genre classification.

On the other hand, in a previous work [5], we sustained that features specifically suited to emotion detection are needed to narrow the so-called semantic gap [12] and their lack hinders the progress of research on MER. In that work, we designed and implemented novel acoustic features, targeting particularly music expressivity and texture, which led to $9 \%$ classification improvement (F1-score). Hence,

- All authors are with the University of Coimbra, Centre for Informatics and Systems of the University of Coimbra, Department of Informatics Engineering. E-mail: \{panda, ruipedro, rsmal\}@dei.uc.pt.

- R. Panda is also with the Polytechnic Institute of Tomar.

- R. Malheiro is also with the Miguel Torga Higher Institute.

\footnotetext{
${ }^{1}$ http://www.music-ir.org/mirex/
}

this study supports the argument that, to further advance the audio MER field, research needs to focus on what we believe is its main, crucial, and current problem: to capture the emotional content conveyed in music through better designed audio features.

This perspective might as well be transversal to most MIR problems, as pointed out in [13], where the authors affirm that "stagnation on most MIR task results is already acknowledged by MIR community". There, the first hypothesis raised is that "MIR approaches should perhaps be more musical knowledge-intensive" since, currently, mostly generic approaches are followed based on "the application of information retrieval solutions for music, without relying on musically meaningful features" [13]. As Pedro Domingos boldly states, "at the end of the day, some machine learning projects succeed and some fail. What makes the difference? Easily the most important factor is the features used" [14].

State-of-the-art solutions are still unable to accurately solve simple problems, such as classification with few emotion classes (e.g., four to five). This is supported by both existing studies $[5,15]$ and the small improvements observed in the 2007-2019 Music Information Retrieval Evaluation eXchange (MIREX) ${ }^{1}$ Audio Mood Classification task, an annual comparison of MER algorithms. There, the best algorithm achieved $69.8 \%$ accuracy in a task comprising five categories. Moreover, this score has remained stable for several years, which calls for methods that help breaking the so-called "glass ceiling" [12].

Given the crucial importance of emotionally-relevant audio features for MER, our goal in this survey is threefold:

- to summarize the most significant knowledge on the relations between music and emotion; this review is structured according to eight musical dimensions (melody, harmony, rhythm, dynamics, tone color, expressivity, texture and form) and sets the ground to identify needs in the design of emotionally-relevant 
audio descriptors;

- $\quad$ to review the current computational audio features that are relevant for MER, particularly the ones available in different open-access audio frameworks, e.g., Marsyas, MIR Toolbox, PsySound and Essentia;

- to unveil possible directions for future research on the topic of feature engineering for MER (based on the above reviews and the identified research needs), as a key effort to break the glass ceiling on audio MER.

Over the years, other authors have offered surveys on Music Emotion Recognition. The most recent we are aware of is the one by Yang et al., from 2018 [15]. Other reviews have been published already several years ago, e.g., the one from 2012 by Yang and Chen [16] or erlier, e.g., [17]. The common characteristic between all of them is that they provide broad MER reviews, tackling topics such as emotion paradigms, approaches for the collection of groundtruth data, types of MER problems (e.g., single-label, multi-label or music emotion variation detection) and overviewing different MER systems. On the contrary, rather than providing a broad but less specific survey, our approach is to offer an updated, deep and specific review on one key MER problem: the design of emotionally-relevant audio features, something that deserved only a somewhat shallow overview in the abovementioned works.

To further clarify the focus of this survey, it is important to mention that approaches based on deep learning techniques are out of the scope of this article, since the breadth of this topic would probably merit a survey in itself. Nevertheless, possible research directions on deep learning for MER are briefly discussed. For the same reason, features based on other modalities, e.g., symbolic or lyrics features, are not covered either. Regarding symbolic features, since some current approaches establish a bridge between the audio and the symbolic MER domains by integrating an audio transcription stage into the feature extraction stage (as discussed in Section 4, e.g., [5]), possible research directions on the exploitation of symbolic features on MER are also briefly discussed.

To summarize, this survey is focused on emotionallyrelevant audio features for MER, covering both low-level (e.g., spectral features, MFCC, etc.), perceptual (e.g., rhythm clarity, modality, articulation, etc.) and high-level semantic features (e.g., genre, danceability, etc.) $[18,19]$.

This paper is organized as follows. Section 2 overviews the relations between music and emotion, which are detailed in Section 3. There, we describe specific associations between each of the eight musical dimensions and different emotions. Section 4 reviews the existing emotionallyrelevant computational audio features, organizing them by musical dimension. Section 5 discusses the gaps and needs to advance the study of audio feature design for MER and points directions for future research. Finally, Section 6 concludes the article.

\section{Music AND Emotion: OVERVIEW}

Music has been with us since prehistoric times, serving as a language to express our emotions. This is regarded as music's primary purpose [20] and the "ultimate reason why humans engage with it" [21].
Our analysis of the relations between music and emotions is structured according to the fundamental musical dimensions usually presented in the musicology literature. Musical dimensions are typically organized into four to eight different categories (depending on the author, e.g., $[22,23])$, each representing a core concept. Here, we employ an eight-category organization comprising: melody, harmony, rhythm, dynamics, tone color (or timbre), expressivity, musical texture and musical form.

The organization of these dimensions is not strict. Many musical features are somehow interconnected and may interact and touch other dimensions. Thus, it can be argued that some of them could be placed in different musical categories. In any case, through this organization, we can understand: i) where features related to emotion belong; ii) which features can be extracted from audio signals with the existing algorithms; iii) and thus, which musical dimensions may lack computational models to extract audio features relevant to emotion.

The relations between music and emotions have been debated for millennia, with associations between modes and emotions found in ancient texts, from Indian, Middle Eastern (e.g., Persian), and far eastern (e.g., Japanese) traditions [21]. Natya Shastra (Nātya Śāstra), an ancient Sanskrit Hindu text describing performance arts, estimated to have been written somewhere between 500 B.C. and 500 A.D. [24] suggests elements such as modes and musical forms as able to express particular emotions.

In ancient Greece, Plato advocated that "good rhythm wait upon good disposition, [...] the truly good and fair disposition of the character and the mind" [25]. In addition, Plato considered harmony as capable of moving the listener, arguing that both "rhythm and harmony find their way to the inmost soul and take strongest hold upon it" [25]. Aristotle supported the same ideas, stating that "rhythms and melodies contain representations of anger and mildness, and also of courage and temperance" [26], while different harmonies could range from relaxing to "violently exciting and emotional" [26].

Scientific studies focusing on the relations between music and emotions started more than a century ago. One of these early examples is a study by Hevner, where the author evaluated the influence of musical factors such as rhythm, pitch, harmony, melody, tempo and mode to each of the eight emotion clusters earlier proposed by her [27]. Along with such studies, music psychologists have proposed different emotion paradigms (e.g., categorical or dimensional) and related taxonomies (e.g., [27, 28]).

Up to this day, this research problem is still far from completely solved. Nevertheless, several contemporary research works had already identified possible correlations or in some cases causal associations between specific musical elements and emotions. One of the most widely accepted is mode: major modes are frequently related to emotional states such as happiness, whereas minor modes are often associated with sadness or anger [29]; simple, consonant, harmonies are usually happy, pleasant or relaxed. On the contrary, complex, dissonant, harmonies relate to emotions such as excitement, tension or sadness, as they create instability in a musical piece [4]. Many other 
musical elements have been related to emotion, namely, e.g., timing, dynamics, articulation, timbre, pitch, interval, melody, harmony, tonality, rhythm, mode, loudness, vibrato or musical form $[4,30]$.

Over the last decades, several associations have been identified, relating specific emotional responses to the musical dimensions described above. The next section details the most relevant findings in this area. For some musical elements, the research can be somewhat contradicting, which can be caused by many factors, from different research methodologies to differences in the scope of the studies (e.g., induced or perceived emotion, significant differences in methodologies, population, and others). This is also caused by the complexity of the topic and indicates that further research is needed.

Most of the associations that we describe below pertain to music emotion perception ${ }^{2}$ or transmission, since most studies tackled that problem. Still, some studies do not clearly state whether their findings concern perceived or induced emotion.

\section{Relations between Musical Dimensions AND EMOTIONS}

In this section we review the known relations between the eight musical dimensions and different emotions.

\subsection{Melody and Emotion}

\section{TABLE 1}

RELATIONS BETWEen MELODIC ELEMENTS AND EMOTIONS.

\begin{tabular}{|c|c|c|}
\hline$M E$ & Value & Associated emotions \\
\hline \multirow[t]{2}{*}{ Pitch } & High & $\begin{array}{l}\text { Surprised, angry, fearful, happy } \\
\text { [33] and others [32]; increased } \\
\text { tense arousal [32] }\end{array}$ \\
\hline & Low & $\begin{array}{l}\text { Sad, bored, pleasant, increased } \\
\text { valence [32]; sad, tender [33] }\end{array}$ \\
\hline \multirow{2}{*}{$\begin{array}{c}\text { Pitch } \\
\text { variation }\end{array}$} & Large & $\begin{array}{l}\text { Happy, active, surprised [32]; } \\
\text { happy [33] }\end{array}$ \\
\hline & Small & $\begin{array}{l}\text { Angry, bored, disgusted [32]; } \\
\text { angry [33] }\end{array}$ \\
\hline \multirow[t]{2}{*}{ Pitch range } & Wide & $\begin{array}{l}\text { Joyful, fearful, scary [32]; happy, } \\
\text { fearful [33] }\end{array}$ \\
\hline & Narrow & Sad [32]; sad, tender [33] \\
\hline \multirow{5}{*}{$\begin{array}{l}\text { Melodic } \\
\text { intervals }\end{array}$} & Large & Powerful [34] \\
\hline & Minor 2nd & Melancholic [34], sad [33] \\
\hline & $\begin{array}{l}\text { Perfect } 4^{\text {th }} \text {, major } \\
6^{\text {th }}, \text { minor } 7^{\text {th }}\end{array}$ & $\begin{array}{l}\text { Carefree [32]; happy (perfect } 4^{\text {th }} \text { ) } \\
\text { [33] }\end{array}$ \\
\hline & Perfect $5^{\text {th }}$ & Carefree, active [32], happy [33] \\
\hline & Octave & Carefree, positive, strong [32] \\
\hline \multirow{2}{*}{$\begin{array}{c}\text { Melodic } \\
\text { direction } \\
\text { and contour }\end{array}$} & Ascending & $\begin{array}{l}\text { Happy, fearful, surprised, angry, } \\
\text { tense [32] }\end{array}$ \\
\hline & Descending & Sad, bored, pleasant [32] \\
\hline \multirow{3}{*}{$\begin{array}{c}\text { Melodic } \\
\text { movement }\end{array}$} & Stepwise motion & Dull melodies [35] \\
\hline & $\begin{array}{l}\text { Intervallic leaps } \\
\text { or skips }\end{array}$ & Exciting melodies [35] \\
\hline & $\begin{array}{l}\text { Stepwise and } \\
\text { skipwise leaps }\end{array}$ & Peaceful melodies [35] \\
\hline
\end{tabular}

\footnotetext{
${ }^{2}$ Emotion in music can be regarded as: i) perceived, as in the emotion an individual identifies when listening; ii) induced or felt, regarding the emotional response a user feels when listening, which can be different from the
}

Melody can be defined as a horizontal succession of pitches (perceptual correlate of fundamental frequency), perceived by listeners as a single musical line.

Given its central role in a musical piece, being (one of) the most memorable elements in a song, associations between melodic cues and emotions are expected and suggested since Plato. Some of the strongest relations are found between wider melodic ranges (pitch ranges) and energetic emotions such as joy [31] or fear [32], while narrow ranges are associated with lower arousal emotions, e.g., sadness, melancholy or tranquility [32]. Other melodic elements, such as ascending versus descending melodic contours, have been studied and related to several emotions [27]. However, some of these are disputed in other studies, arguing that the relation is more complex and involves interactions with other elements such as rhythm and modes [32]. These findings have been observed in cross-cultural studies, where listeners have also associated joy with simpler melodies and sadness with more complex ones [31], even when exposed to unfamiliar tonal systems.

Table 1 summarizes the known relations between melody and emotion. There, ME stands for Musical Element.

\subsection{Harmony and Emotion}

If melody is said to be the horizontal part of music, harmony refers to its "vertical" aspect, i.e., the sound produced by the combination of various pitches (notes or tones) in chords.

\section{TABLE 2}

\begin{tabular}{|c|c|c|}
\hline \multicolumn{3}{|r|}{ ENA HARIVIUTNY AIND EIVIOIIUNS. } \\
\hline$M E$ & Value & Associated emotions \\
\hline \multirow{4}{*}{$\begin{array}{l}\text { Harmonic } \\
\text { perception } \\
\text { (harmonic } \\
\text { intervals) }\end{array}$} & $\begin{array}{l}\text { Consonant } \\
\text { (simple) }\end{array}$ & $\begin{array}{l}\text { Normally associated with positive } \\
\text { emotions, e.g., happy [33], serene and } \\
\text { dignified [27], pleasant, tender [32] }\end{array}$ \\
\hline & $\begin{array}{l}\text { Dissonant } \\
\text { (complex) }\end{array}$ & $\begin{array}{l}\text { Associated mostly with negative } \\
\text { emotions: vigorous, sad [27][33], } \\
\text { unpleasant, tense, fearful, angry [32] }\end{array}$ \\
\hline & High-pitched & Happy, more active/powerful [32] \\
\hline & Low-pitched & Sad, less powerful [32] \\
\hline \multirow{3}{*}{$\begin{array}{l}\text { Harmony } \\
\text { (tonality) }\end{array}$} & Tonal & $\begin{array}{l}\text { In joyful, dull or peaceful melodies, } \\
\text { pleasant [32] }\end{array}$ \\
\hline & Atonal & In angry melodies [32][33] \\
\hline & $\begin{array}{l}\text { Using chromatic } \\
\text { scales }\end{array}$ & In sad and angry melodies [32] \\
\hline \multirow{2}{*}{$\begin{array}{c}\text { Harmony } \\
\text { (mode) }\end{array}$} & Major & $\begin{array}{l}\text { Positive emotions, e.g., happy, } \\
\text { serene, tender [32]; happy [33] }\end{array}$ \\
\hline & Minor & $\begin{array}{l}\text { Negative emotions, e.g., sad, } \\
\text { disgusted and angry [32]; sad [33] }\end{array}$ \\
\hline
\end{tabular}

Harmony, together with rhythm and melody, was thought as able to elicit emotions since ancient times. Consonant harmonies are usually associated with happiness, tranquility, serenity, while dissonant complex harmonies are related with negative emotional states, e.g., tension and sadness, due to the instability they create in the piece [4].

In addition, major modes have been frequently related with positive emotions (e.g., happiness), while minor

perceived one; iii) or transmitted, representing the emotion that the performer or composer aimed to convey [8]. 
modes are linked to negative ones (e.g., sadness) [32]. Some authors such as Cook et al. have tried to further understand this affective response to major/minor chords and resolved/unresolved chords, concluding that this emotional association is "neither due to the summation of interval effects nor simply arbitrary, learned cultural artifacts, but rather that harmony has a psychophysical basis dependent on three-tone combinations" [36].

The relations between harmony and emotion are summarized in Table 2.

\subsection{Rhythm and Emotion}

\section{TABLE 3}

\begin{tabular}{|c|c|c|}
\hline \multicolumn{3}{|c|}{ RELATIONS BETWEEN RHYTHM AND EMOTION. } \\
\hline$M E$ & Value & Associated emotions \\
\hline \multirow[t]{2}{*}{ Tempo } & Fast & $\begin{array}{l}\text { Several, among which happy, } \\
\text { graceful, vigorous, pleasant, } \\
\text { active, angry, fearful, energy } \\
\text { arousal and tension arousal [32]; } \\
\text { happy, anger, fear [33]; high } \\
\text { arousal, e.g., happy, stressful, } \\
\text { amusing [39] }\end{array}$ \\
\hline & Slow & $\begin{array}{l}\text { Several, among which serene, } \\
\text { dreamy, dignified, serious, } \\
\text { tranquil, sentimental, dignified, } \\
\text { sad, peaceful [32]; sad, tender } \\
\text { [33] }\end{array}$ \\
\hline \multirow{3}{*}{$\begin{array}{l}\text { Tempo } \\
\text { and Note } \\
\text { Values }\end{array}$} & $\begin{array}{l}\text { High tempo ( } 150 \\
\text { bpm) and sixteenth } \\
\text { notes }\end{array}$ & $\begin{array}{l}\text { High arousal: happy, amusing, } \\
\text { expressive, stressful [39] }\end{array}$ \\
\hline & $\begin{array}{l}\text { Moderate to fast } \\
\text { tempo (120 or } 150 \\
\text { bpm) and sixteenth } \\
\text { notes }\end{array}$ & Surprised [39] \\
\hline & $\begin{array}{l}\text { Slow to moderate } \\
\text { tempo }(90 \mathrm{bpm}) \text { and } \\
\text { whole and half notes }\end{array}$ & $\begin{array}{l}\text { Sad, boring, relaxing, } \\
\text { expressionless [39] }\end{array}$ \\
\hline \multirow{6}{*}{$\begin{array}{l}\text { Rhythm } \\
\text { Types }\end{array}$} & Regular/smooth & $\begin{array}{l}\text { Happy, glad, serious, dignified, } \\
\text { peaceful, majestic [32]; happy, } \\
\text { anger [33] }\end{array}$ \\
\hline & Irregular/rough & Amusing, uneasy [32] \\
\hline & Complex & Angry [32] [33] \\
\hline & Varied & Joyful [32]; fear [33] \\
\hline & Firm & $\begin{array}{l}\text { Dignified, vigorous, sad, } \\
\text { exciting }^{3} \text { [27], sad [32] }\end{array}$ \\
\hline & Flowing/fluent & $\begin{array}{l}\text { Happy, dreamy, graceful, serene } \\
\text { [27], gay [32] }\end{array}$ \\
\hline \multirow[t]{2}{*}{ Rests } & $\begin{array}{l}\text { After tonal closure (a } \\
\text { sequence which } \\
\text { starts and ends in the } \\
\text { same key) }\end{array}$ & Lower tension [32] \\
\hline & After no tonal closure & $\begin{array}{l}\text { Higher tension than observed if } \\
\text { after tonal closure [32] }\end{array}$ \\
\hline
\end{tabular}

Rhythm represents the element of "time" in music, the patterns of long and short sounds and silences found in music.

\footnotetext{
${ }^{3}$ Sometimes opposite emotions are associated to the same musical element, even in the same study, as found here. In this specific case, Hevner used 142 listeners to associate types of rhythm (firm or flowing) to 8 emotion clusters. Both "sad" and "exciting" clusters were related with firm
}

Rhythm, together with melody and harmony, is one of the dimensions most associated to the emotional expression in music. In fact, some authors consider it the most important one, e.g., [37, 38]. Rhythm elements, such as the augmentation of tempo (from 90 to $150 \mathrm{bpm}$ ), has been shown to increase happiness and surprise measures (i.e., induce) [39], while decreasing sadness. In the study, the authors used two groups of words to study different emotion types: 3 "basic emotions" where users reported what they felt (i.e., induced emotion) on a scale of 1 to 8 ; and 4 "descriptive words" (tension, expressiveness, amusement and attractiveness) to classify (i.e., perceived emotion) the musical piece on a scale of 1 to 5 .

In addition to tempo, the rhythmic unit of a piece has also been shown to influence the emotional message of a song. As an example, variations "of the rhythm of the melody without altering the musical line, harmonics or beat" [39], such as changes from whole and half notes (theme) to eighth or sixteenth, as well syncopated notes, were associated with specific emotions. Similar studies have supported the idea that rhythm is somehow influencing the emotional information in music, e.g., [40].

The associations between rhythm and emotion are summarized in Table 3, based on the reviews presented in [32, $33,41]$, as well as the other mentioned papers.

\subsection{Dynamics and Emotion}

TABLE 4

\begin{tabular}{|c|c|c|}
\hline \multicolumn{3}{|c|}{ RELATIONS BETWEEN DYNAMICS AND EMOTION. } \\
\hline$M E$ & Value & Associated emotions \\
\hline \multirow{2}{*}{$\begin{array}{l}\text { Dynamic } \\
\text { levels } \\
\text { (forte, } \\
\text { piano, etc.) }\end{array}$} & High/Loud & $\begin{array}{l}\text { Excited, triumphant, strong/powerful, } \\
\text { tense, angry, energy arousal and } \\
\text { tension arousal [32]; happy, anger [33] }\end{array}$ \\
\hline & Low/Soft & $\begin{array}{l}\text { Melancholic, peaceful, solemn, fearful, } \\
\text { tender, sad, lower intensity, higher } \\
\text { valence [32]; sad, fear, tender [33] }\end{array}$ \\
\hline \multirow{5}{*}{$\begin{array}{l}\text { Accents } \\
\text { and } \\
\text { changes in } \\
\text { dynamic } \\
\text { levels }\end{array}$} & Large & Fearful [32] [33] \\
\hline & Small & Happy [33], pleasing, active [32] \\
\hline & $\begin{array}{l}\text { Rapid } \\
\text { variations }\end{array}$ & Playful, pleading, fearful [32] \\
\hline & No changes & Sad, peaceful, dignified, happy [32] \\
\hline & $\begin{array}{l}\text { Crescendo, } \\
\text { decrescendo, } \\
\text { accelerando, } \\
\text { ritardando }\end{array}$ & $\begin{array}{l}\text { Said to be useful to describe } \\
\text { perceptual and emotional processes } \\
\text { [44]; anger (accelerando) [33] }\end{array}$ \\
\hline
\end{tabular}

Dynamics represents the variation in loudness or softness of notes in a musical piece.

The influence of dynamics, namely loudness and loudness variations, in music emotions (both induced and perceived) have been studied by some researchers, some of which relate them with specific emotion states. Empirically, an association of loud music (high intensity) with powerful and intense emotions such as joy, anger or tension seems logical. In contrast, soft music is mostly linked to calm, serene or sad music. Such associations have been verified by several researchers [42, 38, 43]. Variations in

rhythm, although the associated weight was lower than the remaining two clusters (dignified and vigorous). 
loudness over a musical piece have also been studied. Namely, larger variations are usually more negative [43], while smaller variations are more positive [32].

Table 4 summarizes the associations between dynamics and emotion.

\subsection{Tone Color and Emotion}

Tone color (or timbre) is related to lower level elements and properties of the sound itself, e.g., amplitude and spectrum, essential to differentiate instruments and voices.

Several sound properties have been associated with emotional states. A rounder amplitude envelope is related with negative emotions such as disgust, sadness or fear [38, 32], while a sharper one gives rise to positive emotions such as happiness or surprise [32], with some authors also linking it to fear [38]. The number of harmonics has also been studied, where a lower number is associated with boredom, happiness or sadness [32], while a high number of harmonics is usually related with emotions with high arousal and negative valence, e.g., anger, disgust, fear [32].

The tone color of specific instruments has also been suspected to carry emotional expression cues. In fact, composers and movie and marketing directors select specific instruments to express distinct emotions. This idea has been supported by studies such as [45]. In this respect, Hailstone et al. state that "timbre (instrument identity) independently affects the perception of emotions in music after controlling for other acoustic, cognitive, and performance factors" [46]. These works highlight the importance of spectral centroid (brightness) as a "significant component in music emotion". Moreover, spectral centroid deviation, spectral shape, attack time and even/odd harmonic ratio were all considered relevant [45].

A summary of the relations between tone color and emotion is presented in Table 5 .

TABLE 5

RELATIONS BETWEEN TONE COLOR (TIMBRE) AND EMOTION.

\begin{tabular}{c|ll}
\multicolumn{1}{c}{ ME } & \multicolumn{1}{c}{ Value } & \multicolumn{1}{c}{ Associated emotions } \\
\hline $\begin{array}{c}\text { Amplitude } \\
\text { envelope }\end{array}$ & Sharp & $\begin{array}{l}\text { Disgusted, bored, potent, fear, } \\
\text { sadness [32] }\end{array}$ \\
$\begin{array}{c}\text { Pleasant, happy, surprised, active, } \\
\text { angry [32]; angry [33] }\end{array}$ \\
\hline $\begin{array}{c}\text { Spectral } \\
\text { envelope (no. } \\
\text { harmonics) }\end{array}$ & Low & $\begin{array}{l}\text { Bored, happy, pleasant, sad [32] } \\
\text { Active, angry, disgusted, fearful, } \\
\text { potent, surprised [32] }\end{array}$ \\
\hline $\begin{array}{c}\text { Spectral } \\
\text { characteristics } \\
\text { (e.g., spectral } \\
\text { centroid, etc.) }\end{array}$ & $\begin{array}{l}\text { Positive } \\
\text { correlation }\end{array}$ & $\begin{array}{l}\text { Positive emotions: happy, heroic, } \\
\text { comic, joyful [45, 47] }\end{array}$ \\
\hline
\end{tabular}

\subsection{Expressivity and Emotion}

Expressive techniques in music encompass several ornaments and features that are used by both composers (to enrich their pieces) and performers (to express their emotions at specific moments). Both parts have been studied and re-

\footnotetext{
${ }^{4}$ From [50], showing only results based on listeners ratings, where significant correlations $(\mathrm{p}<0.05)$ were observed. The indicated associations can be either positive or negatively correlated.
}

lated with specific emotional states. As an example, staccato articulation is normally associated with higher intensity and energetic [32], mostly negative as with fear and anger [38]. On the other hand, legato is associated with softness [32] and sadness [38]. Similar research has been conducted regarding vibratos and emotion expression, observing that "singing an emotional passage influences acoustic features of vibrato when compared with isolated, sustained vowels" [48]. To assess this, classical singers were asked to sing passages of their preference containing both high and low levels of emotion. The analysis of the recordings shows significant changes in vibrato characteristics such as frequency modulation rate and extent.

Regarding emotion expression by the performer, some studies highlighted that artists typically use different ornaments, such as accentuating specific notes considered happy, whereas not doing the same for sadness [49]. In addition, Timmers and Ashley studied the usage by flute and violin performers of specific ornamentations such as trills, turns, mordente, arpeggio and others, when they intended to express one of four specific affect terms (happiness, sadness, anger and love), and how these emotions were perceived by listeners [50]. The accuracy between intended versus rated emotions was lowest for happiness. The performers employed more complex ornamentations for angry and the least complex for sadness.

Table 6 summarizes the main relations between expressivity and emotion.

\section{TABLE 6}

RELATIONS BETWEEN EXPRESSIVITY AND EMOTION.

\begin{tabular}{|c|c|c|}
\hline$M E$ & Value & Associated emotions \\
\hline \multirow[b]{2}{*}{ Articulation } & Legato & Soft [32], tender, sad [32][33] \\
\hline & Staccato & $\begin{array}{l}\text { Intense, energetic, active, } \\
\text { fearful, angry [32]; happy [33] }\end{array}$ \\
\hline \multirow{7}{*}{ Ornamentation ${ }^{4}$} & $\begin{array}{l}\text { Single } \\
\text { appoggiatura } \\
\text { Double } \\
\text { appoggiatura }\end{array}$ & $\begin{array}{l}\text { [pos.] Flute: lovely, sad [50] } \\
\text { [neg.] Flute: happy, angry [50] } \\
\text { [neg.] Violin: sad [50] }\end{array}$ \\
\hline & Trill & $\begin{array}{l}\text { [pos.] Flute: angry [50] } \\
\text { [neg.] Flute: lovely, sad [50] }\end{array}$ \\
\hline & Turn & [pos.] Violin: happy [50] \\
\hline & Mordent & $\begin{array}{l}\text { No significant correlation was } \\
\text { observed [50] }\end{array}$ \\
\hline & Slide & $\begin{array}{l}\text { No significant correlation was } \\
\text { observed [50] }\end{array}$ \\
\hline & Arpeggio & $\begin{array}{l}\text { [pos.] Flute: angry [50] } \\
\text { [neg.] Flute: lovely, sad [50] }\end{array}$ \\
\hline & Substitute & [pos.] Violin: sad [50] \\
\hline \multirow[t]{2}{*}{ Vibrato } & $\begin{array}{l}\text { Higher frequency } \\
\text { modulation (FM) } \\
\text { rate + higher FM } \\
\text { extent + lower } \\
\text { modulation } \\
\text { variability }\end{array}$ & $\begin{array}{l}\text { Observed when classical } \\
\text { singers sang "more emotional } \\
\text { passages"5 (as opposed to } \\
\text { neutral songs) [48]; } \\
\text { Happy (medium-fast rate, } \\
\text { medium extent) [33] }\end{array}$ \\
\hline & $\begin{array}{l}\text { Higher mean } \mathrm{F}_{0}+ \\
\text { higher mean } \\
\text { intensity }\end{array}$ & $\begin{array}{l}\text { Observed in "more emotional } \\
\text { passages" [48] }\end{array}$ \\
\hline
\end{tabular}

${ }^{5}$ As explained earlier, no specific emotions were selected, instead subjects were asked to sing "emotional passages" of their preference and the voice signals were analyzed. 


\subsection{Texture and Emotion}

Musical texture refers to the way the rhythmic, melodic and harmonic information produced by musical instruments and voices is combined in a musical composition. It is thus related to the combination and relations between the musical lines or layers (one or more instruments with the same role) in a song.

Fewer studies have been conducted regarding musical texture and emotions and of these some contain contradicting results. In one of the oldest studies, Kastner and Crowder evaluated the emotional differences between monophonic (melody only) and homophonic textures (melody with block chords accompaniment) by children aged three to twelve. In that study, the unaccompanied version (monophonic) was rated as more positive [51]. A similar result was observed by Webster and Weir, where nonharmonized melodies were considered happier [52]. However, further studies attempting to replicate Kastner and Crowder's findings observed exactly the opposite result. There, not only children but also adult subjects considered monophonic sounds as less happy than accompanied ones [53,54]. A possible explanation to this contradicting results are the different versions of "dense textures" used in each [55], where very basic/simple chords and a single instrument were used in the studies observing negative emotions, while the others used more complex (and thus, with higher density) accompaniments taken from published songbooks. These differences may influence greatly other musical dimensions (e.g., harmony) making it harder to correctly compare the results.

Polyphonic textures, containing several voices, have also been explored recently, suggesting that music with a higher number of voices is perceived as more positive. Such musical excerpts were rated as "sounding more happy, less sad, less lonely, and more proud" [55].

Although further studies are required to better understand exactly how musical texture influences emotion, the existing ones have demonstrated that it can indeed influence emotion in music either directly or by interacting with other features such as tempo and mode [55].

Table 7 summarizes the associations found between musical texture and emotions.

\section{TABLE 7}

\begin{tabular}{|c|c|c|}
\hline \multicolumn{3}{|c|}{ RELATIONS BETWEEN TEXTURE AND EMOTION. } \\
\hline$M E$ & Value & Associated emotions \\
\hline \multirow{2}{*}{$\begin{array}{l}\text { Texture } \\
\text { type }\end{array}$} & Monophonic & $\begin{array}{l}\text { More positive [51] and } \\
\text { happier [52] than homophonic }\end{array}$ \\
\hline & Homophonic & $\begin{array}{l}\text { Happier }[53,54] \text { than } \\
\text { monophonic. }\end{array}$ \\
\hline $\begin{array}{l}\text { Number of } \\
\text { layers and } \\
\text { density }\end{array}$ & $\begin{array}{l}\text { Music with higher } \\
\text { number of voices } \\
\text { (polyphonic) }\end{array}$ & $\begin{array}{l}\text { "more happy, less sad, less } \\
\text { lonely, and more proud" [55] }\end{array}$ \\
\hline
\end{tabular}

\subsection{Form and Emotion}

Musical form or musical structure refers to the overall structure of a musical piece and describes the layout of a composition as divided into sections.

Some studies have investigated possible relations between musical form and emotion. It seems that forms with lower complexity are associated with positive emotions [56] such as relaxation, joy or peace [31]. On the contrary, higher complexity forms usually result in more negative emotions such as sadness [31], which can be higher in arousal (e.g., aggressive) or lower (e.g., melancholy) depending on the dynamism (high or low, respectively) [56].

Some researchers explored the relation between emotion and form by changing the order of sections (in classical music) but no relevant results were obtained [57, 58].

The few associations found between musical form and emotions are presented in Table 8.

TABLE 8

RELATIONS BETWEEN FORM AND EMOTION.

\begin{tabular}{c|ll}
\multicolumn{1}{c}{ ME } & \multicolumn{1}{c}{ Value } & \multicolumn{1}{c}{ Associated emotions } \\
\hline Form & $\begin{array}{l}\text { Positive emotions [56], Joy, } \\
\text { peace, relaxation [31] }\end{array}$ \\
complexity & $\begin{array}{l}\text { High } \\
\text { High complexity and [31] } \\
\text { low dynamism } \\
\text { High complexity and } \\
\text { high dynamism }\end{array}$ & $\begin{array}{l}\text { Depression, melancholy [56] } \\
\text { Aggressiveness, anxiety [56] }\end{array}$ \\
\hline
\end{tabular}

\subsection{Interactions between Musical Dimensions}

As described in the previous sections, each musical element may influence distinct emotional expressions. In fact, emotional content in music is not defined exclusively by a single element but is built by the merging and interaction of several factors. Beyond studying associations concerning musical dimensions and emotions independently, these interactions between several musical dimensions and the associated emotional responses have also been studied and reviewed, e.g., $[59,60]$.

Such works unveil interesting indirect relations and interactions regarding the variation of specific elements and the corresponding emotional changes, as well as possible interactions between elements, resulting in different emotional states. One example is the interaction between tempo and mode [60]: high tempo and minor mode results in only high arousal, while the same high tempo, but with major mode, results in high arousal and positive valence.

Several other authors have studied possible interactions, such as mode and tempo [37], the influence of pitch height, intensity and tempo in valence [42], the influence of rhythm, melodic contour and melodic progression in happy music [32] or interactions between tempo, texture and mode [52].

\section{Computational Audio Features in MER}

In general terms, a feature is a characteristic part of something. Features help to distinguish one thing from another, by providing the essential descriptive primitives by which individual objects or works may be identified [61].

In musical terms, features may be characteristic of a musical work, of a movement, of a composer, of a very specific musical dimension, of a genre, and so forth. As Huron states, "what constitutes a feature depends on the scope of our gaze" [61]. For illustration, features can be employed to represent any aspect that is relevant to the identification of a song, from the chords, to abstract statistics regarding 
physical aspects of the sound wave, rhythm information and others. Summing it up, the goal of feature extraction is to reduce the information of songs to descriptors that can accurately describe them [15].

Over the last decades, several algorithms have been proposed to extract information from audio signals. These features have been developed to solve a myriad of problems, from speech recognition, to content-based retrieval, indexing, and fingerprinting. More recently, a few works studied how the human perception of music characteristics (e.g., tempo) correlates with these audio descriptors, e.g., [62], [63]. It was observed that some features, "in particular those related to loudness, timbre, harmony, and rhythm show high correlations with perceived emotions" [63]. Still, such studies are usually carried with small datasets or specific genres and further research is needed.

Nowadays, most of these feature extraction algorithms are implemented in state-of-the-art audio frameworks, commonly employed in most MIR studies. In this survey, we have reviewed the emotionally-relevant features from 4 common audio frameworks (Marsyas [64], MIR Toolbox (MIR TB) [65], PsySound [66] and Essentia [67]), based on the identified relations between different musical elements and emotions (as discussed in Section 3). The available frameworks vary greatly in many aspects, from userfriendliness to computational efficiency or the number of implemented algorithms. Some are aimed to research, requiring specific environments (e.g., MATLAB), while others are designed with performance in mind, more suited to be used in industry. For an in-depth review, see [68, 69].

In the following, we catalog the audio features that have been proposed in the literature over the years and are now available in these frameworks, organizing them according to the musical dimensions to which they are closest. Besides these frameworks, which implement most of the state-of-the-art audio features, in a recent work, we have contributed with a set of emotionally-relevant audio features, comprising mostly expressivity and musical texture feature [5]. As will be discussed, those features are noticeably underrepresented in the discussed audio frameworks.

Many of the features are extracted repeatedly for smaller excerpts (analysis windows) of the entire audio clip, returning series of data. These frame-level features are usually integrated using statistical moments such as mean, standard deviation, skewness and kurtosis, as well as maximum and minimum, before being used with machine learning techniques.

\subsection{Melody Features}

In this section we describe the audio features that capture information primarily related with melody and its components, as summarized in Table 9.

\section{Pitch}

Pitch represents the perceived fundamental frequency of a sound. It is one of the three major auditory attributes of sounds, along with loudness and timbre. Pitch (as an audio feature) typically refers to the fundamental frequency of a monophonic sound signal and can be calculated using various techniques. One common method to calculate pitch, employed in Marsyas, MIR Toolbox and Essentia is the
YIN algorithm [70]. PsySound3 also implements Swipe and Swipe' algorithms proposed by Camacho [71].

\section{TABLE 9}

MELODY FEATURES.

\begin{tabular}{c|l|l}
\multicolumn{2}{c}{ ME } & \multicolumn{2}{c}{ Feature } & \multicolumn{1}{c}{ Available in } \\
\hline \multirow{3}{*}{ Pitch } & Pitch & $\begin{array}{l}\text { Marsyas, MIR TB, } \\
\text { PsySound3, Essentia } \\
\text { Virtual Pitch Features } \\
\text { Pitch Salience } \\
\text { Predominant Melody F0 } \\
\text { Pitch Content }\end{array}$ \\
\hline Pitch variation & MIDI Note Number stats & Essentia \\
\hline Pitch range & Register Distribution & {$[5]$} \\
\hline Melodic intervals & n.a. & n.a. \\
\hline $\begin{array}{c}\text { Melodic direction } \\
\text { and contour }\end{array}$ & Note Smoothness stats & {$[5]$} \\
\hline Melodic movement & Ratios of Pitch Trans. \\
\hline
\end{tabular}

\section{Virtual Pitch Features}

Ernst Terhardt et al. proposed an algorithm to extract virtual pitch, which is related to the psychoacoustics and modelling of the perceived pitch [72]. The PsySound3 framework implements this algorithm.

\section{Pitch Salience}

The perception of pitch, in particular its salience, is a complex idea that can be roughly explained as how noticeable (that is, strongly marked) is the pitch in a sound, and was proposed as a quick measure of tone sensation. Pure tones have an average pitch salience value close to 0 whereas sounds containing several harmonics in the spectrum have higher salience values. Different approaches have been proposed to extract pitch salience, e.g., [73]. This feature is present in the MIR Toolbox and Essentia.

\section{Predominant Melody FO}

Several authors have proposed algorithms to estimate the fundamental frequency (F0) of the predominant melody in both polyphonic and monophonic music audio signals. This is still an open research problem, and most of the audio frameworks do not include polyphonic audio melody F0 extractors. Still, some of the proposed algorithms are nowadays available as separate tools, e.g., the MELODIA algorithm [73], provided in Essentia.

\section{Pitch content}

Tzanetakis proposed a set of simple features extracted from folded and unfolded pitch histograms (in the folded pitch histogram all notes are mapped to a single octave) to describe pitch information [64]:

- FA0: Amplitude of the maximum peak of the folded histogram;

- UP0: Period of the maximum peak of the unfolded histogram;

- IPO1: Pitch interval between the two most prominent peaks of the folded histogram;

- SUM: The overall sum of the histogram. Although the author described these features in his $\mathrm{PhD}$ 
thesis about the Marsyas framework, the current documentation seems to ignore them. Due to this we could not confirm that the framework is able to extract them.

\section{MIDI Note Number (MNN) statistics}

Panda et al. [5] proposed 6 statistics based on the MIDI note number of each note: MIDImean, i.e., the average MIDI note number of all notes, MIDIstd (standard), MIDIskew (skewness), MIDIkurt (kurtosis), MIDImax (maximum) and MIDImin (minimum).

These features rely on the melody transcription of the original audio waveform. In that work, the authors employed the works by Salamon and Gómez [73] and Dressler [74] to estimate predominant fundamental frequencies as well as saliences. The resulting pitch trajectories are then segmented into individual MIDI notes following the work by Paiva et al. [75].

\section{Register Distribution}

This class of features proposed in [5] indicates how the notes of the predominant melody are distributed across different pitch ranges. Each instrument and voice type have different ranges, which in many cases overlap. The authors selected 6 classes, based on the vocal categories and ranges for non-classical singers. The resulting metrics are the percentage of MIDI note values in the melody that are in each of the following registers: Soprano (C4-C6), Mezzo-soprano (A3-A5), Contralto (F3-E5), Tenor (B2-A4), Baritone (G2-F4) and Bass (E2-E4).

In addition, the authors also propose the register distribution per second, as the ratio of the sum of the duration of notes with a specific pitch range (e.g., soprano) to the total duration of all notes.

\section{Note Smoothness (NS) statistics}

Also related to the characteristics of the melody contour, Panda et al. [5] propose a note smoothness feature as an indicator of how close consecutive notes are, i.e., how smooth is the melody contour. To this end, the difference between consecutive notes (MIDI values) is computed. The usual 6 statistics are also calculated.

\section{Ratios of Pitch Transitions}

In Panda et al. [5], the abovementioned extracted MIDI note values are used to build a sequence of transitions to higher, lower and equal notes.

The obtained sequence marking transitions to higher, equal or lower notes is summarized in several metrics, namely: Transitions to Higher Pitch Notes Ratio, Transitions to Lower Pitch Notes Ratio and Transitions to Equal Pitch Notes Ratio. There, the ratio of the number of specific transitions to the total number of transitions is computed.

\subsection{Harmony Features}

In this section we describe the audio features that capture information primarily related with harmony and its components (Table 10).

\section{Inharmonicity}

The inharmonicity feature is based on number of partials that are not multiples of the fundamental frequency. Inharmonicity influences the timbre perception of a given sound. One approach to compute this was proposed by
Peeters et al. [76] and is implemented in Essentia. The MIR Toolbox measures the inharmonicity as the amount of energy outside the ideal harmonic series, which presupposes that there is only one fundamental frequency [65].

\begin{tabular}{|c|c|c|}
\hline \multirow{2}{*}{\multicolumn{3}{|c|}{ HARMONY FEATURES. }} \\
\hline$M E$ & & Available in \\
\hline \multirow{3}{*}{$\begin{array}{l}\text { Harmonic } \\
\text { perception } \\
\text { (harmonic } \\
\text { intervals) }\end{array}$} & Inharmonicity & MIR TB, Essentia \\
\hline & Chromagram & $\begin{array}{l}\text { Marsyas, MIR TB, } \\
\text { Essentia }\end{array}$ \\
\hline & Chord Sequence & Essentia \\
\hline \multirow{6}{*}{$\begin{array}{l}\text { Harmony } \\
\text { (tonality) }\end{array}$} & Tuning Frequency & Essentia \\
\hline & Key Strength & MIR TB, Essentia \\
\hline & Key and Key Clarity & MIR TB, Essentia \\
\hline & Tonal Centroid Vector & MIR TB \\
\hline & $\mathrm{HCDF}$ & PsySound3 \\
\hline & Sharpness & PsySound3 \\
\hline Harmony (mode) & Modality & MIR TB, Essentia \\
\hline
\end{tabular}

\section{Chromagram}

The chromagram (implemented in Marsyas, MIR Toolbox and Essentia) is used to estimate the energy distribution along pitch classes. It consists of a 12-dimension vector, one for each note, from A to G\# (12 semitone pitch classes), with the respective intensities in each of these classes based on the spectral peaks of the waveform. It is also known as Harmonic Pitch Class Profile (HPCP) [65].

\section{Chord Sequence}

Extracting chords from an audio signal is a complex task, for which researchers have yet to propose robust solutions. The existing methods to estimate this are still experimental, based on pitch class profiles [77]. Essentia implements an algorithm based on this research, able to compute the sequence of chords in a song. Such algorithm calculates the best matching major or minor triad and outputs the result as a string (e.g., A\#, Bm, G\#m, C). The existing implementation is marked as experimental and requires further work before being usable.

\section{Tuning Frequency}

The tuning frequency (available in Essentia) is an estimation of the exact frequency (in $\mathrm{Hz}$ ) on which a song is tuned. It is used as an intermediary step for HPCP calculation and key estimation but can also be applied for classification tasks such as western vs. non-western music [77].

\section{Key Strength}

Key strength (MIR Toolbox and Essentia) consists in the computation of the strength of each possible key candidate to be the key of a given song (e.g., outputting scores between 0 and 1 , or -1 to 1 ). The algorithm is based on the cross-correlation of the chromagram [77].

\section{Key and Key Clarity}

These features (implemented in the MIR Toolbox and Essentia) give a broad estimation of tonal center positions and their respective clarity. This is based on peak picking in the key strength curve. There, the best key(s) is given by the peak abscissa, while the key clarity is the key strength 
associated with the best keys, i.e., the key ordinate [65].

Tonal Centroid Vector (6 dimensions)

In the MIR Toolbox, the tonal centroid is represented as a 6-dimensional feature vector. It corresponds to a projection of the chords along circles of fifths, of minor thirds and of major thirds [78]. It is based on the Harmonic Network or Tonnetz, which is a planar representation of pitch relations, where pitch classes having close harmonic relations such as fifths, major/minor thirds have smaller Euclidean distances on the plane. By calculating the Euclidean distance between successive analysis frames of tonal centroid vectors, the algorithm detects harmonic changes such as chord boundaries from musical audio.

\section{Harmonic Change Detection Function}

PsySound3 implements the Harmonic Change Detection Function (HCDF), which is a method for detecting changes in the harmonic content of musical audio signals proposed by Harte et al. [78]. It can be interpreted as the flux of the tonal centroid, as in the distance between the harmonic regions of successive frames [78].

\section{Sharpness}

Sound can be subjectively rated on a scale from dull to sharp, and sharpness algorithms attempt to model this. PsySound3 implements several algorithms [66], which are essentially weighted centroids of specific loudness.

\section{Modality}

Several algorithms exist to estimate modality, i.e., major vs. minor, returning either a binary label, e.g., major / minor, or a numerical value, e.g., between -1 (minor) and 1 (major) [65]. In the MIR Toolbox and Essentia, the typical strategies use the estimated strength of each key and consist of:

- the difference between the strength of the strongest major and minor keys

- the sum of all the differences between each major key and its relative minor key pair.

\subsection{Rhythm Features}

In this section we describe the audio features that capture information primarily related with rhythm and its components (Table 11).

\section{Beat Spectrum}

The beat spectrum (MIR Toolbox) has been proposed as a measure of acoustic self-similarity as a function of time lag. It is computed from the similarity matrix, obtained by comparing the spectral similarity between all possible pairs of frames from the original audio signal [79].

\section{Beat Location}

Different beat tracking algorithms have been proposed over time. These algorithms estimate the beat locations in an input signal. The Essentia framework implements several beat tracker and rhythm extractor functions, e.g., the multi-feature beat tracker, which extends the idea of measuring the level of agreement between a committee of different beat tracking algorithms in a song-by-song basis [80]. Marsyas implements IBT, a real-time/off-line tempo induction and beat tracking system based on a competing multi-agent strategy that considers parallel hypotheses regarding tempo and beats [81].

TABLE 11

RHYTHM FEATURES.

\begin{tabular}{|c|c|c|}
\hline$M E$ & Feature & Available in \\
\hline \multirow{8}{*}{ Tempo } & Beat Spectrum & MIR TB \\
\hline & Beat Location & Marsyas, Essentia \\
\hline & Onset Time & MIR TB, Essentia \\
\hline & Event Density & MIR TB \\
\hline & Average Duration of Events & MIR TB \\
\hline & Tempo & $\begin{array}{l}\text { Marsyas, MIR TB, } \\
\text { Essentia }\end{array}$ \\
\hline & PLP Novelty Curves & Essentia \\
\hline & HWPS & Marsyas \\
\hline \multirow{5}{*}{$\begin{array}{c}\text { Tempo } \\
\text { and Note } \\
\text { Values }\end{array}$} & Metrical Structure & MIR TB \\
\hline & Metrical Centroid and Strength & MIR TB \\
\hline & Note Duration statistics & [5] \\
\hline & Note Duration Distribution & [5] \\
\hline & Ratios of Note Duration Transitions & {$[5]$} \\
\hline \multirow{3}{*}{$\begin{array}{l}\text { Rhythm } \\
\text { Types }\end{array}$} & Rhythmic Fluctuation & MIR TB \\
\hline & Tempo Change & MIR TB \\
\hline & Pulse / Rhythmic Clarity & MIR TB, Essentia \\
\hline Rests & n.a. & n.a. \\
\hline
\end{tabular}

\section{Onset Time}

Another way of determining the tempo is based on the computation of an onset detection curve, showing the successive bursts of energy corresponding to the successive pulses [76]. Peak picking is automatically performed on the onset detection curve, to show the estimated positions of the note onsets. This feature is provided by the MIR Toolbox and Essentia. In the case of the MIR Toolbox, its onset function is able to return the onset times using any of the following options: peaks, valleys, attack phase and release phase [65].

\section{Event Density}

This feature (MIR Toolbox) estimates the "speed" of a song based on the average number of events in a given time window, i.e., the number of note onsets per second [65].

\section{Average Duration of Events}

In the MIR Toolbox, the duration of events (e.g., a note) can also be estimated from its envelope. One possible approach to estimate this was proposed by Peeters et al. [76]. It consists in detecting attack and release phases and measuring the time (in seconds) between them when the amplitude is at least $40 \%$ of the maximum.

\section{Tempo}

Several algorithms have been proposed to estimate tempo [19], i.e., the speed of a given musical piece, usually indicated in beats per minute (BPM). This feature, available in Marsyas, the MIR Toolbox and Essentia through different alternative algorithms, is typically estimated by detecting periodicities from the onset detection curve [65].

\section{Predominant Local Pulse (PLP) Novelty Curves}

Grosche and Muller introduced a mid-level representation for capturing dominant tempo and predominant local 
pulse even from music with weak non-percussive note onsets and strongly fluctuating tempo [82]. Essentia implements this feature. While the PLP curve does not represent high-level information such as tempo, beat level or location of onset positions, it serves as a tool that may be used for tasks such as beat tracking, tempo and meter estimation.

\section{Harmonically Wrapped Peak Similarity (HWPS)}

Tzanetakis described a set of rhythmic content features calculated with recourse to the Beat Histograms of a song, which proved useful for musical genre classification [64]:

- $\mathrm{A} 0$, A1: relative amplitude of the first (A0), and second (A1) histogram peak;

- RA: ratio of the amplitude of the second peak divided by the amplitude of the first peak;

- P1, P2: Period of the first and second peak in BPM;

- SUM: histogram sum (indication of beat strength) Subsequently, HWPS, a feature following similar principles has been proposed and integrated into Marsyas to calculate harmonicity by taking "into account spectral information in a global manner" [83].

\section{Metrical Structure}

This feature provides a detailed description of the hierarchical metrical structure by detecting periodicities from the onset detection curve and tracking a broad set of metrical levels [65]. This extractor is used to calculate the meterbased tempo estimation in the MIR Toolbox.

\section{Metrical Centroid and Strength}

These functions provide two descriptors derived from the above metrical analysis performed in the MIR Toolbox:

- Dynamic metrical centroid: estimation of the metrical activity, based on the computation of the centroid of the selected metrical level [65];

- Dynamic metrical strength: an indicator of the clarity and strength of the pulsation. Estimates whether a "clear and strong pulsation, or even a strong metrical hierarchy is present", or if the opposite is true, where "the pulsation is somewhat hidden, unclear" [65] or a complex mix of pulsations.

\section{Note Duration statistics}

Panda et al. propose note duration statistics (the same six ones, as proposed for the melody dimension), based on the duration of each note [5].

\section{Note Duration Distribution}

Moreover, note duration distribution features are also proposed in [5]: Short Notes Ratio, Medium Length Notes Ratio and Long Notes Ratio. Similarly, the authors compute the note duration distribution per second, for each of the three duration classes defined.

\section{Ratios of Note Duration Transitions}

Finally, Panda et al. also propose ratios of note duration transitions [5], namely, Transitions to Longer Notes Ratio, Transitions to Shorter Notes Ratio and Transitions to Equal Length Notes Ratio.

\section{Rhythmic Fluctuation}

This feature (present in the MIR Toolbox) estimates the rhythm content of an audio signal. This estimation is based on spectrogram computation transformed by auditory modeling followed by spectrum estimation in each band [84], i.e., the rhythmic periodicity along auditory channels.

\section{Tempo Change}

An indicator of tempo change over time is estimated by computing the difference between successive values of the tempo curve in the MIR Toolbox. This feature is expressed independently from the choice of a metrical level by computing the ratio of tempo values between successive frames and is expressed in logarithmic scale (base 2) [65].

\section{Pulse / Rhythmic Clarity}

This feature (implemented in the MIR Toolbox and Essentia) estimates the "rhythmic clarity", an indicator of the clarity and strength found in the beats estimated by tempo estimation algorithms. Distinct heuristics exist to this estimation. The most common uses the autocorrelation curve that is computed during tempo estimation [65]. Essentia computes an approximate metric calling it beats loudness.

\subsection{Dynamics Features}

In this section we describe the audio features that capture information primarily related with dynamics and its components (Table 12).

\section{TABLE 12}

DYNAMICS FEATURES.

\begin{tabular}{|c|c|c|}
\hline$M E$ & Feature & Available in \\
\hline \multirow{10}{*}{$\begin{array}{c}\text { Dynamic } \\
\text { levels (forte, } \\
\text { piano, etc.) }\end{array}$} & RMS Energy & $\begin{array}{l}\text { Marsyas, MIR TB, } \\
\text { Essentia }\end{array}$ \\
\hline & Low Energy Rate & Marsyas, MIR TB \\
\hline & Sound Level & PsySound3 \\
\hline & $\begin{array}{c}\text { Instantaneous Level, Freq. } \\
\text { and Phase }\end{array}$ & PsySound3 \\
\hline & Loudness & PsySound3, Essentia \\
\hline & Timbral Width & PsySound3 \\
\hline & Volume & PsySound3 \\
\hline & Sound Balance & MIR TB, Essentia \\
\hline & Note Intensity statistics & {$[5]$} \\
\hline & Note Intensity Distribution & [5] \\
\hline \multirow{2}{*}{$\begin{array}{c}\text { Accents and } \\
\text { changes in } \\
\text { dynamic } \\
\text { levels }\end{array}$} & $\begin{array}{c}\text { Ratios of Note Intensity } \\
\text { Transitions }\end{array}$ & [5] \\
\hline & $\begin{array}{c}\text { Crescendo and Decrescendo } \\
\text { metrics }\end{array}$ & [5] \\
\hline
\end{tabular}

\section{Root-Mean-Square (RMS) Energy}

The RMS energy (implemented in Marsyas, the MIR Toolbox and Essentia) is used to measure the power of a signal over a window, or global energy. This is usually computed by taking the root-mean-square (RMS) [64]. It roughly describes the loudness of a musical signal.

\section{Low Energy Rate}

Low energy rate (available in Marsyas and the MIR Toolbox) measures the percentage of frames with lessthan-average energy [64]. This metric estimates the temporal distribution of energy, in order to understand if this energy remains constant between frames or if some frames are more contrastive than others.

\section{Sound Level}

This descriptor (present in PsySound3) corresponds to the 
power sum of the spectrum for each time window, expressed in decibel. At a higher level, when appropriately calibrated, this represents the unweighted sound pressure level of the signal in each analysis window [66].

\section{Instantaneous Level, Frequency and Phase}

These features (implemented in PsySound3) consist in applying a Hilbert transform to the audio waveform, resulting in three different outputs: the instantaneous level, instantaneous frequency and instantaneous phase. The instantaneous level can be regarded as the sound pressure level derived from the Hilbert transform [66].

\section{Loudness}

Sound loudness is the subjective perception of the intensity of a sound. This metric is measured in sones, where a doubling in sones corresponds to a doubling of loudness [66]. Several loudness metrics have been proposed over the years, which are available in PsySound3 and Essentia.

\section{Timbral Width}

Timbral width (PsySound3) is one of six measures of timbre proposed by Malloch in a method called loudness distribution analysis [85]. Timbral width can be regarded as "a measure of the fraction of loudness that lies outside of the loudest band, relative to the total loudness" [85].

\section{Volume}

Volume refers roughly to the perceived "size" of the sound, or the auditory volume of pure tones. This concept was first studied by Stevens [86] and, later on, Cabrera [87] developed a computational volume model for arbitrary spectra, which was integrated into PsySound3. In his work, Cabrera proposes two diotic volume models. The first uses a weighted ratio between the binaural loudness and sharpness, which is the specific loudness centroid on the Bark scale. A second and better performing model uses a simpler centroid to overcome limitations in the method of sharpness calculation selected by the authors [87].

\section{Sound Balance}

Sound balance can be estimated through the Maximum Amplitude Position to Total Envelope Length Ratio (MaxToTotal and MinToTotal), provided in the MIR Toolbox and Essentia. This is a metric to understand how much the maximum amplitude (peak) in a sound envelop is off the center. To this end, the ratio between the index of the maximum (or minimum) value of the envelope of a signal and the total length of the envelope is computed. If the peak amplitude is found close to the beginning (e.g., decrescendo sounds), this ratio will be close to 0 . A value of 0.5 means that the peak is close to the middle and near 1 if at the end of the sound (e.g., crescendo sounds) [69].

\section{Note Intensity statistics}

Panda et al. compute the usual 6 statistics based on the median pitch salience of each note [5].

\section{Note Intensity Distribution}

In addition, Panda et al., 2018 propose note intensity distribution features [5]. This class of features indicates how the notes of the predominant melody are distributed across three intensity ranges, leading to the following features: Low Intensity Notes Ratio, Medium Intensity Notes Ratio and High Intensity Notes Ratio. The same features are also computed per second.

\section{Ratios of Note Intensity Transitions}

Panda et al., 2018 also propose ratios of Note Intensity Transitions: Transitions to Higher Intensity Notes Ratio, Transitions to Lower Intensity Notes Ratio and Transitions to Equal Intensity Notes Ratio [5].

\section{Crescendo and Decrescendo (CD) metrics}

Panda et al. identify notes as having crescendo or decrescendo based on the intensity difference between the first and the second half of the note [5]. From these, the authors compute the number of crescendo and decrescendo notes (per note and per second). In addition, they compute sequences of notes with increasing or decreasing intensity, computing the number of sequences for both cases (per note and per sec) and the length of crescendo sequences in notes and in seconds, using the 6 usual statistics.

\subsection{Tone Color Features}

In this section we describe the audio features that capture information primarily related with tone color (timbre) and its components (Table 13).

\section{TABLE 13}

TONE COLOR (TIMBRE) FEATURES.

\begin{tabular}{|c|c|c|}
\hline$M E$ & Feature & Available in \\
\hline \multirow{4}{*}{$\begin{array}{c}\text { Amplitude } \\
\text { envelope }\end{array}$} & Attack/Decay Time & MIR TB, Essentia \\
\hline & Attack/Decay Slope & MIR TB \\
\hline & Attack/Decay Leap & MIR TB \\
\hline & Zero Crossing Rate & Marsyas, MIR TB, Essentia \\
\hline \multirow{5}{*}{$\begin{array}{c}\text { Spectral } \\
\text { envelope (no. } \\
\text { harmonics) }\end{array}$} & Spectral Flatness & Marsyas, MIR TB, Essentia \\
\hline & Spectral Crest Factor & Marsyas \\
\hline & Irregularity & MIR TB \\
\hline & Tristimulus & Essentia \\
\hline & $\begin{array}{c}\text { Odd-to-even } \\
\text { harmonic energy } \\
\text { ratio }\end{array}$ & Essentia \\
\hline \multirow{16}{*}{$\begin{array}{c}\text { Spectral } \\
\text { characteristics } \\
\text { (e.g., spectral } \\
\text { centroid) }\end{array}$} & Spectral Centroid & $\begin{array}{l}\text { Marsyas, MIR TB, } \\
\text { PsySound3, Essentia }\end{array}$ \\
\hline & Spectral Spread & MIR TB, PsySound3, Essentia \\
\hline & Spectral Skewness & MIR TB, PsySound3, Essentia \\
\hline & Spectral Kurtosis & MIR TB, PsySound3, Essentia \\
\hline & Spectral Entropy & MIR TB, Essentia \\
\hline & Spectral Flux & Marsyas, MIR TB, Essentia \\
\hline & Spectral Rolloff & Marsyas, MIR TB, Essentia \\
\hline & $\begin{array}{l}\text { High-frequency } \\
\text { Energy }\end{array}$ & MIR TB, Essentia \\
\hline & $\begin{array}{c}\text { Cepstrum } \\
\text { (Real/Complex) }\end{array}$ & PsySound3 \\
\hline & $\begin{array}{c}\text { Energy in } \\
\text { Mel/Bark/ERB Bands }\end{array}$ & MIR TB, PsySound3, Essentia \\
\hline & MFCCs & Marsyas, MIR TB, Essentia \\
\hline & LPCCs & Marsyas, Essentia \\
\hline & Linear Spectral Pairs & Marsyas \\
\hline & Spectral Contrast & Essentia \\
\hline & Roughness & MIR TB, PsySound3, Essentia \\
\hline & $\begin{array}{l}\text { Spectral and Tonal } \\
\text { Dissonance }\end{array}$ & PsySound3 \\
\hline
\end{tabular}




\section{Attack/Decay Time}

One of the aspects influencing tone color is the sound envelope, which can be divided into four parts: attack, decay, sustain and release. Several descriptors can be extracted from it, mostly related with the attack phase, i.e., from the starting point of the envelope until the amplitude peak is attained. One of these descriptors is the attack time (present in the MIR Toolbox and Essentia), which consists in the estimation of temporal duration of the various attack phases in an audio signal [76]. The MIR Toolbox is also able to compute the decay time.

\section{Attack/Decay Slope}

The attack slope (available in the MIR Toolbox) is another descriptor extracted from the attack phase [76]. It consists on the estimation of the average slope of the entire attack phase, since its start to the peak. The MIR Toolbox is also able to extract the same information from the decay phase, related to its decrease slope [65].

\section{Attack/Decay Leap}

The attack leap is a simple descriptor related to the attack phase. In the MIR Toolbox, it consists in the estimation of the amplitude difference between the beginning (bottom) and the end (peak) of the attack phase [65]. As with the previous features, the MIR Toolbox outputs a similar descriptor related with the decay phase.

\section{Zero Crossing Rate (ZCR)}

The Zero Crossing Rate (Marsyas, MIR Toolbox Essentia) represents the number of times the waveform changes sign in a window (crosses the $x$-axis). It can be used as a simple indicator of change of frequency or noisiness. As an example, heavy metal music, due to guitar distortion and heavy percussion, will tend to have much higher zero crossing values than classical music [64]. Sometimes the ZCR derivative is also computed, representing the absolute value of the window-to-window change in zero crossing rate.

\section{Spectral Flatness}

The spectral flatness (Marsyas, MIR Toolbox, Essentia) indicates whether the spectrum distribution is smooth or spiky, i.e., estimates to which degree the frequencies in a spectrum are uniformly distributed (noise-like) [65]. It is usually computed as the ratio between the geometric mean and the arithmetic mean [76]. Marsyas adopts a different approach, proposed in [88], calculating the spectral flatness in different spectral bands.

\section{Spectral Crest Factor (SCF)}

The spectral crest factor [88] is a measure of the "peakiness" of a spectrum and is inversely proportional to the spectral flatness measure. It is commonly used to distinguish noiselike from tone-like sounds due to their different spectral shapes, where noise-like sounds have lower spectral crests. In Marsyas, the SCF is computed as the ratio of the maximum and mean spectrum powers of a subband.

\section{Irregularity}

Irregularity, also known as spectral peaks variability, is the degree of variation of the amplitude of successive spectral peaks [65]. This feature is present in the MIR Toolbox.

\section{Tristimulus}

The tristimulus feature [76], implemented in Essentia, quantifies the relative energy of partial tones by three parameters that measure the energy ratio of the first partial (tristimulus1), second, third and fourth partials (tristimulus2) and the remaining (tristimulus3).

\section{Odd-to-even Harmonic Energy Ratio}

The odd-to-even harmonic energy (Essentia) ratio "distinguishes sounds with predominant energy at odd harmonics (such as clarinet sounds) from other sounds with smoother spectral envelopes (such as the trumpet)" [76].

\section{Spectral Moments: Centroid, Spread, Skewness and Kurtosis}

The four spectral moments (implemented in the MIR Toolbox, PsySound and Essentia) are useful measures of spectral shape [76]. The spectral centroid (also available in Marsyas) is the first moment (mean) of the magnitude spectrum of the short-time Fourier Transform (STFT).

The spectral spread represents the standard deviation of the magnitude spectrum. Thus, it is a measure of the dispersion or spread of the spectrum.

Spectral skewness is the third central moment of the magnitude spectrum and it is a measure of its symmetry.

Finally, in simple terms, spectral kurtosis, or the fourth central moment of the magnitude spectrum, captures information about existing outliers.

\section{Spectral Entropy}

The spectral entropy of a signal is a measure of its spectral power distribution, based on Shannon entropy [89] from the information theory field. This feature is implemented in the MIR Toolbox and Essentia.

\section{Spectral Flux}

Spectral flux (Marsyas, MIR Toolbox, Essentia) is a measure of the amount of spectral change in a signal, i.e., the distance between the spectra of successive frames [64]. Spectral flux has also been shown by user experiments to be an important perceptual attribute in the characterization of the timbre of musical instruments [90].

\section{Spectral Rolloff}

Spectral rolloff (Marsyas, MIR Toolbox, Essentia) is often used as an indicator of the skewness of the frequencies present in a window. According to Tzanetakis [64], the spectral rolloff is defined as the frequency $R \_t$ below which $85 \%$ of the magnitude distribution is concentrated. The percentage varies among authors, but $85 \%$ is the current default value for most frameworks.

\section{High-frequency Energy}

Several algorithms have been proposed to estimate the high-frequency content in a signal. Brightness (also called high-frequency energy) is one of such algorithms, implemented in the MIR Toolbox. This typically consists in fixing a minimum frequency value and measuring the amount of energy above that frequency [65]. The Essentia framework implements a different algorithm, named highfrequency content (HFC), to measure the amount of highfrequency energy from the signal spectrum. HFC is computed by applying one of the several algorithms, e.g., [91]. 


\section{Cepstrum (Real / Complex)}

The cepstrum is the result of taking the inverse Fourier transform of the logarithm of the estimated spectrum of a signal [92]. It can be regarded as a measure of the rate of change in the different spectral bands. Cepstral analysis has applications in fields such as pitch analysis, echo detection and human speech processing, by providing a simple way to separate formants (due to filtering in the vocal tract) from the vocal source [93]. Cepstral analyzers are available in PsySound3.

\section{Energy in Mel/Bark/ERB Bands}

In audio signal processing, it is often important to decompose the original signal into a series of audio signals of different frequencies (i.e., low to high-frequency channels), enabling the study of each channel separately. This is inspired by the human cochlea, which can be regarded as a filter bank, distributing the frequencies into critical bands. Several scales have been proposed, each one using a particular range of frequencies, e.g., the Mel, Bark or Equivalent rectangular bandwidth (ERB) scales [94]. The energy in the Mel/Bark bands is computed in the MIR Toolbox and in Essentia. The energy in the ERB bands is computed in the same two frameworks, as well as PsySound3.

\section{Mel-Frequency Cepstral Coefficients (MFCC)}

MFCCs [95] are another measure of spectral shape. The frequency bands are positioned logarithmically on the Mel scale and cepstral coefficients are then computed based on the Discrete Cosine Transform of the log magnitude spectrum. Typically, only the first 13 cepstral coefficients are usually returned by audio frameworks. These 13 coefficients are mostly used for speech representation but Tzanetakis states that "the first five coefficients are adequate for music representation" [64]. This descriptor is provided by Marsyas, the MIR Toolbox and Essentia.

\section{Linear Predictive Coding Coefficients (LPCC)}

Linear predictive coding is used in speech research to represent the spectral envelope of a digital speech signal in compressed form, using to this end information of a linear predictive model [96]. LPCCs, available in Marsyas and Essentia, represent the cepstral coefficients derived from linear prediction and have been used in a wide range of speech applications, such as speech analysis, encoding and speech emotion recognition [96].

\section{Linear Spectral Pairs (LSP)}

Linear Spectral Pairs (available in Marsyas) are an alternative representation of linear prediction coefficients (LPC) for transmission over a channel. LSPs have several properties (e.g., smaller sensitivity to quantization noise) that make them superior to direct quantization of LPCs. Thus, LSPs are useful in speech recognition and coding [97].

\section{Spectral Contrast}

The octave-based spectral contrast is a feature proposed by Jiang et al. [98] to represent the spectral characteristics of an audio signal, specifically the relative spectral distribution. According to the authors, the feature has been tested in music type classification problems, demonstrating a "better discrimination among different music types than mel-frequency cepstral coefficients (MFCC)" [98], which is one of the features typically used in such problems. It is implemented in Essentia.

\section{Roughness (Sensory Dissonance)}

Sensory dissonance, also known as roughness, is related to the beating phenomenon that occurs whenever a pair of sinusoids are close in frequency [99]. This feature is implemented in Marsyas, the MIR Toolbox and Essentia using different algorithms, the method by Sethares, which estimates total roughness by averaging all dissonance estimates across all possible peak pairs of the spectrum [100].

\section{Spectral and Tonal Dissonance}

PsySound3 computes spectral and tonal dissonance features. Dissonance measures the harshness or roughness of the acoustic spectrum [66]. The dissonance generally implies a combination of notes that sound harsh or are unpleasant to people when played at the same time. PsySound3 provides two descriptions of acoustic dissonance: "spectral dissonance" which uses all Fourier components, and "tonal dissonance" which uses a peak extraction algorithm before calculating dissonance.

\subsection{Expressivity Features}

In this section we describe the audio features that capture information primarily related with expressiveness. As will be observed, we are only aware of one feature of this type in the analyzed audio frameworks. Hence, we have recently proposed a set of novel features targeting expressivity features [5]. Table 14 summarizes the available expressivity features.

\begin{tabular}{c|c|l}
\multicolumn{3}{c}{ TABLE 14 } \\
\multicolumn{1}{c}{ EXPRESSIVITY FEATURES. } \\
\multicolumn{1}{c|}{ Feature } & \multicolumn{1}{c}{ Available in } \\
\hline \multirow{2}{*}{ Articulation } & Average Silence Ratio & MIR TB \\
& Articulation metrics & {$[5]$} \\
\hline \multirow{2}{*}{ Ornamentation } & Glissando metrics & {$[5]$} \\
& Portamento metrics & {$[101]$} \\
\hline Vibrato & Vibrato metrics & {$[5,101,102]$} \\
\hline Tremolo & Tremolo metrics & {$[5]$} \\
\hline
\end{tabular}

\section{Average Silence Ratio (ASR)}

Average Silence Ratio is a feature proposed by Feng et al. as an estimation for articulation [3]. It is defined as the ratio of silence frames in one-second time windows. According to the author "lower ASR means fewer silence frames present in musical piece, or legato in articulation, and the higher ASR means more silence frames present in musical piece, or staccato in articulation". This feature is implemented in the MIR Toolbox.

\section{Articulation metrics}

Articulation is a technique affecting the transition or continuity between notes or sounds. Panda et al. [5] proposed an approach to detect legato (i.e., connected notes played "smoothly") and staccato (i.e., short and detached notes). Based on their algorithm, all the transitions between notes in the song clip are classified and, from them, several metrics are extracted such as ratio of staccato, legato and other transitions and longest sequence of each articulation type.

\section{Glissando metrics}

Glissando is another kind of expressive articulation, which 
consists in the glide from one note to another. It is used as an ornamentation, to add interest to a piece and thus may be related to specific emotions in music. Panda et al. [5] proposed a glissando detection algorithm based on which several glissando features are extracted, e.g., glissando presence, extent, duration, direction, slope and glissando to non-glissando ratio (i.e., the ratio of notes containing glissando to the total number of notes).

\section{Portamento metrics}

Computational models of portamento, the smooth and monotonic increase or decrease in pitch from one note to the next, were proposed in [101] by using Hidden Markov Models in the vibrato-free pitch curve (flatten out).

\section{Vibrato metrics}

Vibrato is an expressive technique used in vocal and instrumental music that consists in a regular oscillation of pitch. Its main characteristics are the amount of pitch variation (extent) and the velocity (rate) of this pitch variation. Panda et al. [5] proposed a vibrato detection algorithm based on the analysis of F0 sequence of each note, from which several features are extracted, e.g., vibrato presence, rate, extent, coverage, high-frequency coverage, vibrato to non-vibrato ratio and vibrato notes base frequency. Other approaches to extract vibrato parameters were proposed, such as using filter diagonalization methods [101] or directly from the spectrogram using predefined vibrato templates [102].

\section{Tremolo metrics}

Tremolo is a trembling effect, somewhat similar to vibrato but regarding change of amplitude. Although, in the survey presented in Section 3, we have not found any relations between tremolo and emotion, we decided to extract a number of tremolo metrics, based on a tremolo detection algorithm, similar to our vibrato detection approach [5]. There, the sequence of pitch saliences of each note is used instead of the F0 sequence, since tremolo represents a variation in intensity or amplitude of the note. Several tremolo features are extracted, e.g., tremolo presence, rate, extent, coverage, and tremolo to non-tremolo ratio.

\subsection{Texture Features}

In this section we describe the audio features that capture information primarily related with musical texture. To the best of our knowledge, none of the features studied or found in the analyzed audio frameworks are primarily related with musical texture. As such, we have recently proposed a set of novel musical texture features in [5], where the sequence of multiple frequency estimates was employed to measure the number of simultaneous layers in each frame of the entire audio signal, leading to the features summarized in Table 15 and described below.

TABLE 15

TEXTURE FEATURES.

\begin{tabular}{c|c|c}
\multicolumn{1}{c}{ ME } & Feature & Available in \\
\hline $\begin{array}{c}\text { Number of layers } \\
\text { and density }\end{array}$ & Musical Layers statistics & {$[5]$} \\
& Musical Layers Distribution & {$[5]$} \\
Ratio of Musical Layers Transitions & {$[5]$} \\
\hline Texture type & n.a. & n.a. \\
\hline
\end{tabular}

\section{Musical Layers statistics}

Panda et al. proposed musical layer statistics [5]. There, the number of multiple F0s are estimated from each frame of the song clip. The number of layers in a frame is defined as the number of obtained multiple F0s in that frame. Then, the 6 usual statistics regarding the distribution of the number of musical layers across frames were computed.

\section{Musical Layers Distribution}

Additionally, in [5] the number of F0 estimates in a given frame is divided into four classes: i) no layers; ii) a single layer; iii) two simultaneous layers; iv) and three or more layers. The percentage of frames in each class is computed.

\section{Ratio of Musical Layers Transitions}

Panda et al. [5] proposed these features to capture information about the changes from a specific musical layer sequence to another. They employ the number of different fundamental frequencies in each frame, identifying consecutive frames with distinct values as transitions and normalizing the total value by the length of the audio segment (in secs). In addition, they also compute the length in seconds of the longest segment for each musical layer.

\subsection{Form Features}

In this section we describe the audio features that capture information primarily related with musical form. Extracting musical form and structure information directly from the audio signal is more difficult when compared to other lower level features (e.g., spectral/timbral statistics). Thus, few computational extractors are available today, as presented in Table 16 and described below.

\section{TABLE 16}

FORM FEATURES.

\begin{tabular}{|c|c|c|}
\hline \\
\hline$M E$ & Feature & Available in \\
\hline Form Complexity & Structural Change & [103] \\
\hline \multirow{2}{*}{ Organization Levels } & Similarity Matrix & MIR TB \\
\hline & Novelty Curve & MIR TB \\
\hline Song Elements & Higher-Level Form Analysis & [104-106] \\
\hline
\end{tabular}

\section{Structural Change}

The amount of change of various underlying basis features at different time intervals, combined into a meta-feature, correlates with the human perception of complexity in music [103]. The typical implementation uses chroma, rhythm and timbre information and exclusively aims at discovering the quantity of change, illustrating it with a visual audio flower plot [103].

\section{Similarity Matrix}

Some approaches estimate musical structure based on the similarity between adjacent segments or frames [65]. These similarities are often represented using an inter-frame or inter-segment similarity matrix, showing the differences between all possible pairs of frames from the input audio signal. The similarity matrix computation uses a specific set of frame statistics (e.g., spectral features) and a distance function, to calculate the proximity between each pair of frames. As an example, the MIR Toolbox can use MFCCs, key strength, tonal centroid, chromagram and others with one of several distance functions. 


\section{Novelty Curve}

Based on the specific musical characteristics of each segment or frame, obtained for instance with a similarity matrix, a novelty curve can be obtained by comparing the successive frames to estimate temporal changes in the song [65]. In this novelty curve, implemented in the MIR Toolbox, the probability of transitioning to a different state over time is represented by the curve peaks.

\section{Higher-level (HL) Form Analysis}

Modeling the fundamental aspects of musical sections in a unified way to identify song elements such as intro, bridge or chorus is still and open problem. Some of the most promising approaches apply higher-level solutions combining low-level features, statistics and machine learning. These include hierarchical semi-markov models [104], convex non-negative matrix factorization, spectral clustering [105] and deep learning [106].

\subsection{Vocal Features}

A few works have studied emotion in speaking and singing voice [107], as well as the related acoustic features [108]. In fact, "using singing voices alone may be effective for separating the "calm" from the "sad" emotion, but this effectiveness is lost when the voices are mixed with accompanying music" and "source separation can effectively improve the performance" [15].

To this end, Panda et al. [5] applied the singing voice separation approach proposed by Fan et al. [109] (although separating the singing voice from accompaniment in an audio signal is still an open problem) and the Voice Analysis Toolkit, a "set of Matlab code for carrying out glottal source and voice quality analysis" 6 to extract the features summarized in Table 17 and described below.

\begin{tabular}{c|c}
\multicolumn{2}{c}{ TABLE 17 } \\
VOCAL FEATURES. \\
Feature & \multicolumn{2}{c}{ Available in } \\
\hline All Features from the Vocals Channel & {$[5]$} \\
Voiced and Unvoiced statistics & {$[5]$} \\
Creaky Voice statistics & {$[5]$}
\end{tabular}

\section{All Features from the Vocals Channel}

Besides extracting features from the original audio signal, Panda et al. [5] also extracted the previously described features from the signal containing only the separated voice.

\section{Voice and Unvoiced statistics}

In [5], the authors also proposed statistics related to the amount of voiced and unvoiced sections in a song. These include, among others, the number of voice segments, the mean, maximum, minimum, standard deviation, kurtosis and skewness of the duration of voice segments, as well as the number of voice segments per second.

\section{Creaky Voice statistics}

Panda et al. [5] computed statistics related with the presence of creaky voice, "a phonation type involving a low frequency and often highly irregular vocal fold vibration, [which] has the potential [...] to indicate emotion" [110].

\subsection{High-Level Features}

Finally, frameworks such as the MIR Toolbox and Essentia provide a few experimental higher-level features, related with complex concepts such as emotion, genre or danceability. Most, if not all, of these are predictors, combining classification algorithms and previously gathered data to label the source audio files into a fixed set of tags. A summary of these predictors is presented in Table 18 and listed below.

TABLE 18

HIGH-LEVEL FEATURES.

\begin{tabular}{c|l} 
Feature & \multicolumn{1}{c}{ Available in } \\
\hline Emotion & MIR Toolbox, Essentia \\
Classification-based Feat. (genre, etc.) & Essentia \\
Danceability & Essentia \\
Dynamic Complexity & Essentia
\end{tabular}

\section{Emotion}

The MIR Toolbox extracts an emotion descriptor based on the analysis of the audio content of a given recording. The output is given in two distinct paradigms: a categorical approach comprising 5 emotions and a 3-dimensional space composed of activity (energetic arousal), valence (pleasure-displeasure continuum) and tension (tense arousal).

The classification process is based on the work by Eerola et al. [111] and uses multiple linear regression with the 5 best performing predictors. Given its reliance on previously established weights, this extractor is only reliable in the MIR Toolbox version (v1.3) where it was initially "calibrated". Newer versions output "distorted results" [65].

The Essentia library implements a similar feature, classifying songs in 4 distinct emotions. It contains pre-trained models and requires the Gaia library to apply similarity measures and classifications on the extracted features [67].

\section{Classification-based Features (genre, etc.)}

In a similar way to the emotion descriptor extractor (or predictor), Essentia also includes Gaia trained models for [67]:

- musical genre (using 4 different databases)

- ballroom music classification

- western / non-western music

- tonal / atonal

- danceability

- voice / instrumental

- gender (male / female singer)

- timbre classification

These musical descriptors work as a typical classification problem, by extracting a set of features from the source audio signals and feeding them to classification models trained with them in other datasets.

The genre feature is particularly relevant for music emotion recognition since some emotions are frequently associated with specific genres, as concluded by Laurier [4]. The author used automatic genre classification to improve his previous emotion classification results.

\section{Danceability}

As opposed to the aforementioned danceability extractor built as a pre-trained classification model, Streich proposed a low-level audio feature derived from Detrended 
Fluctuation Analysis (DFA) to characterize audio signals in terms of its danceability [112].

\section{Dynamic Complexity}

Streich also studied the automated estimation of the complexity of music based on the musical audio signal, proposing a set of complexity descriptors [112]. The proposed algorithms focus on aspects of acoustics, rhythm, timbre, and tonality. The Essentia library implements an extractor to estimate dynamic complexity, or whether a song contains a high dynamic range. This descriptor consists in the average absolute deviation from the global loudness level estimate on the $\mathrm{dB}$ scale.

\section{Discussion and Research DiRECTIONS}

\subsection{Feature Analysis along Musical Dimensions}

Table 19 presents the number of described features per musical dimension.

TABLE 19

NUMBER OF AUDIO DESCRIPTORS PER MUSICAL DIMENSION.
\begin{tabular}{lcc} 
Musical dimension & Number of features & Percentage of total \\
\hline Melody & 9 & $10.6 \%$ \\
Harmony & 10 & $11.8 \%$ \\
Rhythm & 16 & $18.8 \%$ \\
Dynamics & 12 & $14.1 \%$ \\
Tone Color & 25 & $29.4 \%$ \\
Expressivity & 6 & $7.1 \%$ \\
Texture & 3 & $3.5 \%$ \\
Form & 4 & $4.7 \%$ \\
\hline Total & 85 & $100 \%$
\end{tabular}

As abovementioned, many of these features are framelevel features, which are normally integrated using statistical moments. This increases the final number of descriptors to several hundred [5] and is especially true for tone color features, where some features divide the audio signal in bands and output time-series data (e.g., MFCCs). As such, and based on the figures in Table 19, we conclude that the number of available audio features is very unbalanced across musical dimensions. Musical texture, expressivity and form are especially lacking, in contrast to tone color, which is the most represented category, mostly due to the large set of spectral features available (centroid, etc.). In [5], we have contributed to reduce that imbalance by proposing emotionally-relevant features, particularly for the expressivity and texture dimensions.

The low number of texture, form and expressivity features is not a surprise. We believe this is caused by two main reasons: i) on the one hand, the difficulty to create robust algorithms to capture such music elements; ii) on the other hand, the lack of music psychology studies on the relations between emotion and those dimensions, which could drive the creation of computational models.

Regarding the analysis of the importance of specific features to emotion recognition, few studies have addressed this issue in a systematic way, e.g., [5]. There, the conducted analysis, based on Russell's emotion quadrants [28], suggested that tone color features (particularly spectral features) dominated all quadrants, possibility due to their prevalence (as discussed above). Nevertheless, texture features were in the top 5 for quadrant 2 (anxiety quadrant, or Q2) and proved relevant for Q1 (happiness), as well, helping to improve the classification performance of the proposed algorithm. Vibrato was also an important feature for Q2. As for Q3 (depression), besides tonal features, texture, inharmonicity and tremolo also proved relevant, along with vocal features. Finally, dynamics, texture and expressivity features (namely, vibrato) were important to discriminate Q4 (contentment).

Besides the lack of texture, form and expressivity features, "more features are needed to better discriminate Q3 from Q4, which musically share some common characteristics such as lower tempo, less musical layers and energy, use of glissandos and other expressive techniques" [5]. Thus, in the next section we discuss research directions to advance the state-of-the-art in the creation of novel emotionally-relevant features for each musical dimension.

\subsection{Novel Audio Futures: Research Directions}

\section{Form}

Regarding computational models of form complexity, we are only aware of one work, which might work as a surrogate of musical complexity [103]. Higher-level features to capture form types from audio are still missing and some recent works have been attacking the problem with higher level solutions, e.g., employing machine learning to identify elements such as verse and chorus [104-106].

The impact of other elements of form on emotion, e.g., organizational levels (passage, piece, cycle) or song elements (introduction, chorus, bridges, etc.), should be further researched by the music psychology community, despite a few computational models found in the literature that might partially capture such information (e.g., similarity matrix and novelty curve).

\section{Texture}

The texture dimension, as abovementioned, requires further music psychology studies to better understand how it influences emotion. Nevertheless, the features we proposed in [5] proved relevant, namely the number of musical layers in the recognition of happy music.

These features only approximate the actual number of layers in a song, hence more advanced computational models are needed, probably requiring robust source separation and instrument recognition in polyphonic music. This is an active research problem (e.g., [113]), with great advances in the last years due to the application of deep learning models, as is the case of the Spleeter library, able to perform various types of separation (e.g., vocals, accompaniment, drums, bass, and others) [114].

Tackling this problem would also serve the creation of algorithms for the detection of texture types (monophonic, homophonic, polyphonic) and density (thin, thick), for which no computational models are known (see Table 15).

\section{Expressivity}

Regarding expressivity, the music psychologic community has offered important inputs to understand its impact on emotion. Yet, despite our contributions with several artic- 
ulation (staccato and legato), glissando, vibrato and tremolo metrics, this dimension still lacks computational models, particularly for the detection of ornamentations other than glissando and portamento (see Tables 6 and 14). Also, the algorithms we proposed were only indirectly evaluated through their impact on emotion classification, and so ground truth data on those problems is needed.

\section{Melody}

As for the other musical dimensions, music psychology researchers have provided a great amount of knowledge that could be further exploited to create computational models that capture such musical elements.

Starting with melody, most melodic elements are reasonably covered, as summarized in Table 9. However, features for melodic intervals are still missing. Moreover, further computational features related to melodic movement, direction and contour should be developed. As with many other problems in Music Information Retrieval, problems such as full or melody transcription are still open, which limits the accuracy of current MER systems that rely on them. This also applies to computational models of the dimensions discussed below (e.g., tonality and rhythm).

\section{Harmony}

As for harmony, all elements with emotional relevance have computational features to capture them (Table 10): harmonic perception (e.g., inharmonicity), tonality (e.g., tonal centroid vector) and mode (e.g., modality).

\section{Rhythm}

Regarding rhythm, although most rhythmic elements are reasonably covered this dimension is missing computational features that capture rest characteristics (Table 11). Still, higher-level audio features that capture the types of rhythm (regular, irregular, complex, fluent, etc.) are still missing (see Tables 3 and 11).

\section{Dynamics}

As for dynamics, all elements have associated features (Table 12). Still, computational models to detect the types of dynamic levels (forte, piano, etc.) would be beneficial.

\section{Tone Color}

The tone color dimension is also reasonably well covered, particularly regarding spectral characteristics (see Table 13). Still, as with musical texture, tone color would also benefit from accurate instrument recognition in polyphonic context. Moreover, this dimension would also benefit from higher-level features on the types of amplitude envelope (e.g., round, sharp).

\section{Vocal Features}

As for vocal features, with the recent advances in areas such as source separation, as previously described, new paths should be explored. For instance, additional features that proved useful for speech emotion should be taken into consideration [16]. Moreover, the idea can be extended, e.g., by further separating the accompaniment and analyzing each layer in isolation, since they may sometimes carry different emotional information [15]. This can be complemented with genre or even lyrical information (natural language processing) and integrated with a meta-classifier.

\subsection{Deep Learning Perspectives}

Finally, besides the classical handcrafted feature engineering approach, deep learning/feature learning techniques have attracted great attention in the last years. The most notable example is the resurgence of neural network techniques, specifically deep learning, to a myriad of problems, fueled by the improvements in computer processing (e.g., using graphic processing units). Several MER studies have already employed techniques such as convolutional and recurrent neural networks [10].

Despite (so far) slight improvements in classification accuracy, such approaches raise several points that must be considered. First, to fully exploit the potential of deep learning solutions, massive amounts of good quality data are required. Unfortunately, the creation of large MER datasets have been known to be problematic due to the associated subjectivity and complexity of data collection [5]. Hence, strategies to obtain sizeable and good quality data for audio MER are a key need.

Also, deep learning models are opaque in the sense that the extracted features are often difficult to interpret, which hinders the possibility to acquire novel knowledge regarding the relations between emotions and the extracted features. In fact, "although deep neural networks have exhibited superior performance in various tasks, interpretability is always [the] Achilles' heel" of such approaches, despite a few efforts to address it, as surveyed in [115]. Hence, interpretability issues in deep neural networks are another important problem to tackle in the future.

\subsection{Audio-based Symbolic Features}

As discussed, some approaches establish a bridge between the audio and the symbolic MER domains by integrating an audio transcription stage into the feature extraction stage. Hence, the approached followed in [5] can be further exploited by integrating symbolic (MIDI) features that are available in several frameworks, e.g., MIDI Toolbox or jSymbolic (cited in [2]).

\section{CONCLUSION}

This article offered a comprehensive review of the current emotionally-relevant computational audio features. This survey was supported by the music psychology literature on the relations between eight musical dimensions (melody, harmony, rhythm, dynamics, tone color, expressivity, texture and form) and specific emotions. From this review, we concluded that computational audio features able to capture elements of musical form, texture and expressivity are especially needed to break the current glass ceiling in MER, as shown in [5]. Moreover, the development of such computational tools would benefit from further music psychology studies, particularly regarding the actual impact of musical form and texture on emotion. We believe this article opens several research lines to expand the state-ofthe-art on Music Emotion Recognition.

\section{ACKNOWLEDGMENT}

This work was supported by the MERGE project financed by Fundação para Ciência e a Tecnologia (FCT) - Portugal. 


\section{REFERENCES}

[1] D. Huron, "Perceptual and cognitive applications in music information retrieval," Cognition, vol. 10, no. 1, pp. 83-92, 2000.

[2] R. Panda, R. Malheiro, B. Rocha, A. Oliveira, and R.P. Paiva, "Multi-Modal Music Emotion Recognition: A New Dataset, Methodology and Comparative Analysis," Proc. 10th Int. Symp. Comput. Music Multidisciplinary Res. - CMMR'2013, 2013.

[3] Y. Feng, Y. Zhuang, and Y. Pan, "Popular Music Retrieval by Detecting Mood," Proc. 26th Annu. Int. ACM SIGIR Conf. Res. Dev. Inf. Retr., vol. 2, no. 2, pp. 375-376, 2003.

[4] C. Laurier, "Automatic Classification of Musical Mood by Content-Based Analysis," Pompeu Fabra Univ., 2011.

[5] R. Panda, R. Malheiro, and R.P. Paiva, "Novel Audio Features for Music Emotion Recognition," IEEE T. Affect. Comput., 2018.

[6] T. Li and M. Ogihara, "Detecting emotion in music," Proc. 4th Int. Soc. of Music Inf. Retrieval Conf. (ISMIR 2003), 2003.

[7] B. Wu, E. Zhong, A. Horner, and Q. Yang, "Music Emotion Recognition by Multi-label Multi-layer Multi-instance Multiview Learning,". Proc. 22th ACM Int. Conf. on Multimedia, 2014.

[8] Y.-H. Yang, Y.-C. Lin, Y.-F. Su, and H.H. Chen, "A Regression Approach to Music Emotion Recognition," IEEE T. Audio Speech Lang. Processing, vol. 16, no. 2, pp. 448-457, 2008.

[9] R. Malheiro, R. Panda, P. Gomes, and R.P. Paiva, "EmotionallyRelevant Features for Classification and Regression of Music Lyrics," IEEE T. Affect. Comput., vol. 9, no. 2, pp. 240-254, 2018.

[10] M. Malik et al., "Stacked Convolutional and Recurrent Neural Networks for Music Emotion Recognition," Proc. Sound and Music Comp. (SMC'2017), 2017.

[11] A. Aljanaki, Y.-H. Yang, and M. Soleymani, "Developing a benchmark for emotional analysis of music," PLoS One, vol. 12, no. 3, 2017.

[12] Ò. Celma, P. Herrera, and X. Serra, "Bridging the Music Semantic Gap," Workshop on Mastering the Gap: From Inf. Extraction to Semantic Representation, 2006.

[13] R. Scholz, G. Ramalho, and G. Cabral, "Cross Task Study on MIREX Recent Results: an Index for Evolution Measurement and Some Stagnation Hypotheses," Proc. 17th Int. Soc. of Music Inf. Retrieval Conf. (ISMIR 2016), 2016.

[14] P. Domingos, "A few useful things to know about machine learning," Comm. ACM, vol. 55, no. 10, pp. 78-87, 2012.

[15] X. Yang, Y. Dong, and J. Li, "Review of data features-based music emotion recognition methods," Multimed. Syst., vol. 24, 2018.

[16] Y.-H. Yang and H.H. Chen, "Machine Recognition of Music Emotion: A Review," ACM Trans. Intell. Syst. Technol., no. 3, 2012.

[17] A. Huq, J.P. Bello and R. Rowe, "Automated Music Emotion Recognition: A Systematic Evaluation," J. New Music Res., vol. 39, no. 3, pp. 227-244, 2010.

[18] A. Friberg, E. Schoonderwaldt, A. Hedblad, M. Fabiani and A. Elowsson, "Using listener-based perceptual features as intermediate representations in music information retrieval," $J$. Acoust. Soc. Am., vol. 136, no. 4, pp. 1951-1963, 2014.

[19] A. Elowsson and A. Friberg, "Tempo estimation by modelling perceptual speed", Music Inf. Retrieval Eval. Ex. (MIREX), 2013.

[20] D. Cooke, The language of music. Oxford Univ. Press, 1959.

[21] A. Pannese, M.-A. Rappaz, and D. Grandjean, "Metaphor and music emotion: Ancient views and future directions," Consciousness and Cognition, vol. 44, pp. 61-71, 2016.

[22] L.B. Meyer, Explaining Music: Essays and Explorations. Univ. of California Press, 1973.

[23] H. Owen, Music theory resource book. Oxford Univ. Press, 2000.
[24] W. Dace (1963), "The Concept of "Rasa" in Sanskrit Dramatic Theory," Educational Theatre J., vol. 15, no. 3, p. 249-254.

[25] Plato, "Republic III," Plato in Twelve Volumes, vols. 5-6. Cambridge, MA: Harvard Univ. Press, (375 B.C.), 1969.

[26] Aristotle, "Politics". Aristotle in 23 Volumes, vol. 21. Cambridge, MA: Harvard Univ. Press, (IV c B.C., 1944).

[27] K. Hevner, "Experimental Studies of the Elements of Expression in Music," Am. J. Psychol., vol. 48, no. 2, pp. 246-268, 1936.

[28] J.A. Russell, "A circumplex model of affect," J. Pers. Soc. Psychol., vol. 39, no. 6, pp. 1161-1178, 1980.

[29] A. Gabrielsson and E. Lindström, "The Influence of Musical Structure on Emotional Expression," Music and Emotion, vol. 8, Oxford Univ. Press, pp. 223-248, 2001.

[30] A. Friberg, "Digital Audio Emotions - An Overview of Computer Analysis and Synthesis of Emotional Expression in Music," Proc. 11th Int. Conf. on Digital Audio Effects (DAFx), pp. 1-6, 2008.

[31] L.-L. Balkwill and W.F. Thompson, "A Cross-Cultural Investigation of the Perception of Emotion in Music: Psychophysical and Cultural Cues," Music Perception, vol. 17, no. 1, pp. 43-64, 1999.

[32] A. Gabrielsson and E. Lindström, "The Role of Structure in the Musical Expression of Emotions," Handbook of Music and Emotion: Theory, Research, Applications, P.N. Juslin and J.A. Sloboda, eds., Oxford Univ. Press, pp. 367-400, 2011.

[33] P.N. Juslin and P. Laukka, "Expression, Perception, and Induction of Musical Emotions: A Review and a Questionnaire Study of Everyday Listening," J. New Music Res., vol. 33, no. 3, pp. 217-238, 2004.

[34] T.F. Maher and D.E. Berlyne, "Verbal and Exploratory Responses to Melodic Musical Intervals," Psychol. of Music, vol. 10, no. 1, pp. 11-27, 1982.

[35] W.F. Thompson and B. Robitaille, "Can Composers Express Emotions through Music?," Empirical Stud. of the Arts, vol. 10, no. 1, pp. 79-89, 1992.

[36] N.D. Cook and T.X. Fujisawa, "The Psychophysics of Harmony Perception: Harmony is a Three-Tone Phenomenon," Empirical Musicology Review, vol. 1, no. 2, pp. 106-126, 2006.

[37] L. Gagnon and I. Peretz, "Mode and tempo relative contributions to "happy-sad" judgements in equitone melodies," Cognition $\mathcal{E}$ Emotion, vol. 17, no. 1, pp. 25-40, 2003.

[38] P.N. Juslin, "Perceived Emotional Expression in Synthesized Performances of a Short Melody: Capturing the Listener's Judgment Policy," Music. Sci., vol. 1, no. 2, pp. 225-256, 1997.

[39] A. Fernández-Sotos, A. Fernández-Caballero, and J.M. Latorre, "Influence of Tempo and Rhythmic Unit in Musical Emotion Regulation," Frontiers in Comp. Neuroscience, vol. 10, no. 80, 2016.

[40] M. Plewa and B. Kostek, "A Study on Correlation between Tempo and Mood of Music," Proc. 133th Audio Eng. Soc. Conv. (AES 133), 2012.

[41] P.N. Juslin and R. Timmers, "Expression and Communication of Emotion in Music Performance," Handbook of Music and Emotion: Theory, Research, Applications, P. N. Juslin and J.A. Sloboda, eds., Oxford Univ. Press, pp. 452-489, 2011.

[42] G. Ilie and W.F. Thompson, "A Comparison of Acoustic Cues in Music and Speech for Three Dimensions of Affect," Music Perception, vol. 23, no. 4, pp. 319-330, 2006.

[43] K.B. Watson, "The nature and measurement of musical meanings," Psychological Monographs, vol. 54, i-43, 1942.

[44] S.K. Langer, Philosophy in a New Key: A Study in the Symbolism of Reason, Rite, and Art. Harvard Univ Press, 1957. 
[45] B. Wu, A. Horner, and C. Lee, "The Correspondence of Music Emotion and Timbre in Sustained Musical Instrument Sounds," J. Audio Engineering Soc., vol. 62, no. 10, pp. 663-675, 2014.

[46] J.C. Hailstone, R. Omar, S.M.D. Henley, C. Frost, M.G. Kenward, and J.D. Warren, "It's not what you play, it's how you play it: timbre affects perception of emotion in music," Quarterly J. of Exp. Psychol., vol. 62, no. 11, pp. 2141-2155, 2009.

[47] B. Wu, A. Horner, and C. Lee, “Musical timbre and emotion: The identification of salient timbral features in sustained musical instrument tones equalized in attack time and spectral centroid," Proc. 40th Int. Computer Music Conf. - ICMC 2014, 2014.

[48] C. Dromey, S.O. Holmes, J.A. Hopkin, and K. Tanner, "The Effects of Emotional Expression on Vibrato," J. of Voice, vol. 29, no. 2, pp. 170-181, 2015.

[49] E. Lindström, "Expression in Music: Interaction between Performance and Melodic Structure," Meeting of the Soc. for Music Percept. and Cognition, 1999.

[50] R. Timmers and R. Ashley, "Emotional Ornamentation in Performances of a Handel Sonata," Music Percept., vol. 25, no. 2, pp. 117-134, 2007.

[51] M.P. Kastner and R.G. Crowder, "Perception of the Major/Minor Distinction: IV. Emotional Connotations in Young Children," Music Percept., vol 8, no. 2, pp. 189-201, 1990.

[52] G. D. Webster and C. G. Weir, "Emotional Responses to Music: Interactive Effects of Mode, Texture, and Tempo," Motivation and Emotion, vol. 29, no. 1, pp. 19-39, 2005.

[53] A. H. Gregory, L. Worrall, and A. Sarge, "The development of emotional responses to music in young children," Motivation and Emotion, vol. 20, no. 4, pp. 341-348, 1996.

[54] R. McCulloch, "Modality and children's affective responses to music," Undergraduate project for the Perception and Performance course (Ian Cross, instructor), 1999.

[55] Y. Broze, B.T. Paul, E.T. Allen, and K.M. Guarna, "Polyphonic Voice Multiplicity, Numerosity, and Musical Emotion Perception," Music Percept., vol. 32, no. 2, pp. 143-159, 2014.

[56] M. Imberty, Understanding Music: Phychological Music Semantics (Entendre la Musique: Sémantique Psychologique de la Musique). Dunod, 1979.

[57] V.J. Konečni and M.P. Karno, "Empirical investigations of the hedonic and emotional effects of musical structure," Musikpsychologie, vol. 11, 119-137, 1994.

[58] B. Tillmann and E. Bigand, "Does Formal Musical Structure Affect Perception of Musical Expressiveness?," Psychol. of Music, vol. 24, no. 1, pp. 3-17, 1996.

[59] A. Gabrielsson and P.N. Juslin, "Emotional expression in music," Handbook of Affective Sciences (Series in Affective Science), R.J. Davidson eds., Oxford Univ. Press, pp. 503-534, 2003.

[60] E. Schubert, "Measurement and Time Series Analysis of Emotion in Music," PhD Diss., School of Music and Music Education, Univ. of New South Wales, 1999.

[61] D. Huron, "What is a Musical Feature? Forte's Analysis of Brahms's Opus 51, No. 1, Revisited," The Online J. of the Soc. for Music Theory, vol. 7, no. 4, 2001.

[62] A. Rodà, S. Canazza, and G. De Poli, “Clustering affective qualities of classical music: Beyond the valence-arousal plane," IEEE Trans. Affect. Comput., vol. 5, no. 4, pp. 364-376, 2014.

[63] M. Schedl, E. Gomez, E. S. Trent, M. Tkalcic, H. Eghbal-Zadeh, and A. Martorell, "On the Interrelation between Listener Characteristics and the Perception of Emotions in Classical Orchestra Music," IEEE Trans. Affect. Comput., vol. 9, no. 4, pp. 507, 2018.
[64] G. Tzanetakis, "Manipulation, Analysis and Retrieval Systems for Audio Signals," PhD diss., Princeton Univ., 2002.

[65] O. Lartillot, MIR Toolbox 1.7.1 User's Manual. Oslo, Norway: Univ. of Oslo, 2018.

[66] D. Cabrera, S. Ferguson, and E. Schubert, “'Psysound3': Software for Acoustical and Psychoacoustical Analysis of Sound Recordings," Proc. 13th Int. Conf. on Auditory Display, 2007.

[67] D. Bogdanov et al., "ESSENTIA: An audio analysis library for music information retrieval," Proc. 14th Int. Soc. of Music Inf. Retrieval Conf. (ISMIR 2013), 2013.

[68] D. Moffat, D. Ronan, and J.D. Reiss, "An Evaluation of Audio Feature Extraction Toolboxes," Proc. 18th Int. Conf. on Digital Audio Effects (DAFx-15), 2015.

[69] R. Panda, "Emotion-based Analysis and Classification of Audio Music," PhD diss., Univ. of Coimbra, 2019.

[70] A. de Cheveigné and H. Kawahara, "YIN, a fundamental frequency estimator for speech and music," J. Acoust. Soc. Am., vol. 111, no. 4, pp. 1917-1930, 2002.

[71] A. Camacho, "SWIPE: A Sawtooth Waveform Inspired Pitch Estimator," PhD Diss., Univ. of Florida, 2007.

[72] E. Terhardt, G. Stoll, and M. Seewann, "Algorithm for extraction of pitch and pitch salience from complex tonal signals," J. Acoust. Soc. Am., vol. 71, no. 3, pp. 679-688, 1982.

[73] J. Salamon and E. Gómez, "Melody Extraction From Polyphonic Music Signals Using Pitch Contour Characteristics," IEEE Trans. Audio. Speech. Lang. Process., vol. 20, no. 6, pp. 1759-1770, 2012.

[74] K. Dressler, "Automatic Transcription of the Melody from Polyphonic Music," PhD Diss., Ilmenau Univ. of Technol., 2016.

[75] R. P. Paiva, T. Mendes, and A. Cardoso, "From Pitches to Notes: Creation and Segmentation of Pitch Tracks for Melody Detection in Polyphonic Audio", J. of New Music Res., vol. 37, no. 3, 2008.

[76] G. Peeters, B.L. Giordano, P. Susini, N. Misdariis, and S. McAdams, "The Timbre Toolbox: Extracting audio descriptors from musical signals.", J. Acoust. Soc. Am., vol. 130, no. 5, 2011.

[77] E. Gómez, "Tonal Description of Music Audio Signals," Pompeu Fabra Univ., 2006.

[78] C. Harte, M. Sandler, and M. Gasser, "Detecting harmonic change in musical audio," Proc. 1st ACM Workshop on Audio and Music Computing Multimedia (AMCMM'06), 2006.

[79] J.T. Foote, M.L. Cooper, and U. Nam, "Audio retrieval by rhythmic similarit," Proc. 3rd Int. Conf. on Music Inf. Retr., 2002.

[80] J. Zapata, M.E.P. Davies, and E. Gómez, "Multi-Feature Beat Tracking," IEEE/ACM Trans. on Audio, Speech, and Language Process., vol. 22, no. 4, pp. 816-825, 2014.

[81] J.L. Oliveira, F. Gouyon, L.G. Martins, and L.P. Reis, "IBT: A Real-Time Tempo and Beat Tracking System," Proc. 11th Int. Soc. for Music Inf. Retrieval Conf. (ISMIR 2010), 2010.

[82] P. Grosche and M. Müller, “A Mid-Level Representation for Capturing Dominant Tempo and Pulse Information in Music Recordings," Proc. 10th Int. Soc. for Music Inf. Retr. Conf. , 2009.

[83] M. Lagrange, L.G. Martins, and G. Tzanetakis, "A Computationally Efficient Scheme for Dominant Harmonic Source Separation," Proc. IEEE Int. Conf. on Acoustics, Speech and Signal Process. (ICASSP 2008), 2008.

[84] E. Pampalk, A. Rauber, and D. Merkl, "Content-based organization and visualization of music archives," Proc. 10th ACM Int. Conf. on Multimedia (ACM MM 2002), 2002.

[85] S.N. Malloch, "Timbre and Technology: an analytical partnership," PhD Diss., Univ. of Edinburgh, 1997.

[86] S.S. Stevens, "The Volume and Intensity of Tones," Am. J. 
Psychol., vol. 46, no. 3, pp. 397-408, 1934.

[87] D. Cabrera, "The Size of Sound: Auditory Volume Reassessed, “ Proc. 1999 Australasian Computer Music Association Conf., 1999.

[88] E. Allamanche, O. Hellmuth, B. Fröba, T. Kastner, \& M. Cremer, "Content-based Identification of Audio Material Using MPEG-7 Low Level Description," Proc. 2nd Int. Symp. on Music Inf. Retrieval (ISMIR 2001), 2001.

[89]C.E. Shannon, "A mathematical theory of communication," Bell Syst. Tech. J., vol. 27, no. 3, pp. 379-423, 1948.

[90] J.M. Grey, "An Exploration of Musical Timbre," PhD Diss., Stanford Univ., 1975.

[91] P. Masri and A. Bateman, "Improved modelling of attack transients in music analysis-resynthesis," Proc. Int. Comp. Music Conf. (ICMC 1996), 1996.

[92] B. P. Bogert, J. R. Healy, and J. W Tukey, “The Quefrency Alanysis (sic) of Time Series for Echoes: Cepstrum, PseudoAutocovariance, Cross-Cepstrum, and Saphe Cracking," Proc. Symp. Time Series Analysis, pp. 209-243, 1963.

[93] A. M. Noll, "Cepstrum Pitch Determination," J. Acoust. Soc. Am., vol. 41, pp. 2, 293-309, 1967

[94] J. Harrington and S. Cassidy, Techniques in Speech Acoustics. Dordrecht: Kluwer Academic Publishers, 1999.

[95] S. Davis and P. Mermelstein. "Comparison of parametric representations for monosyllabic word recognition in continuously spoken sentences," IEEE T Acoust. Speech, vol. 28, no. 4, pp. 357-366, 1980.

[96] M. El Ayadi, M.S. Kamel, and F. Karray, "Survey on Speech Emotion Recognition: Features, Classification Schemes, and Databases, " Pattern Recognition, vol. 44, no. 3, pp. 572-587, 2011.

[97] F. Zheng, Z. Song, L. Li, W. Yu, and W. Wu, "The distance measure for line spectrum pairs applied to speech recognition," Proc. 5th Int. Conf. Spoken Language Process. (ICSLP'98), 1998.

[98] D.-N. Jiang, L. Lu, H.-J. Zhang, J.-H. Tao, and L.-H. Cai, “Music type classification by spectral contrast feature," Proc IEEE Int. Conf. Multimedia and Expo (ICME 2002), 2002.

[99] R. Plomp and W.J.M. Levelt, "Tonal Consonance and Critical Bandwidth," J. Acoust. Soc. Am., vol. 38, no. 4, pp. 548-560, 1965.

[100]W.A. Sethares, Tuning, Timbre, Spectrum, Scale. Springer, 1998.

[101] L. Yang, K. Z. Rajab, and E. Chew, “AVA: An interactive system for visual and quantitative analyses of vibrato and portamento performance styles," Proc. 17th Int. Soc. for Music Inf. Retrieval Conf. (ISMIR 2016), 2016.

[102] J. Driedger, S. Balke, S. Ewert, and M. Müller, "Template-based vibrato analysis of music signals," Proc. 17th Int. Soc. for Music Inf. Retrieval Conf. (ISMIR 2016), 2016.

[103] M. Mauch and M. Levy, "Structural change on multiple time scales as a correlate of musical complexity," Proc. 12th Int. Soc. Music Inf. Retr. Conf. ISMIR 2011, pp. 489-494, 2011.

[104] G. Shibata, R. Nishikimi, E. Nakamura, and K. Yoshii, “Statistical Music Structure Analysis Based on a Homogeneity-, Repetitiveness-, and Regularity-Aware Hierarchical Hidden Semi-Markov Model," in Proc. of the 20th Int. Soc. Music Inf. Retr.Conf., 2019.

[105] B. McFee and D. P. W. Ellis. Analyzing song structure with spectral clustering. Int. Soc. for Music Inf. Retrieval Conf., 2014.

[106] K. Ullrich, J. Schlüter, and T. Grill, "Boundary detection in music structure analysis using convolutional neural networks," Proc. 15th Int. Soc. Music Inf. Retr. Conf., pp. 417-422, 2014.

[107]K.R. Scherer, J. Sundberg, L. Tamarit, and G.L. Salomão,
"Comparing the acoustic expression of emotion in the speaking and the singing voice," Comput. Speech Lang., vol. 29, no. 1, pp. 218-235, 2015.

[108]F. Eyben, G.L. Salomão, J. Sundberg, K.R. Scherer, and B.W. Schuller, "Emotion in the singing voice - a deeperlook at acoustic features in the light ofautomatic classification," EURASIP J. Audio, Speech, Music Process., vol. 2015, no. 19, 2015.

[109]Z.-C. Fan, J.-S. R. Jang, and C.-L. Lu, “Singing Voice Separation and Pitch Extraction from Monaural Polyphonic Audio Music via DNN and Adaptive Pitch Tracking," Proc. IEEE 2nd Int. Conf. Multimedia Big Data (BigMM), 2016.

[110]A. Cullen, J. Kane, T. Drugman, and N. Harte, “Creaky Voice and the Classification of Affect," Proc. Workshop in Affect. and Social Speech Signals (WASSS), 2013.

[111]T. Eerola, O. Lartillot, and P. Toiviainen, "Prediction of multidimensional emotional ratings in music from audio using multivariate regression models," Proc. 10th Int. Soc. for Music Inf. Retrieval Conf. (ISMIR 2009), 2009.

[112]S. Streich, "Music Complexity: a Multi-Faceted Description of Audio Content," PhD Diss., Pompeu Fabra Univ., 2007.

[113]S. Gurunani, M. Sharma, A. Lerch, "An Attention Mechanism for Musical Instrument Recognition," Proc. 20th Int. Soc. for Music Inf. Retrieval Conf. (ISMIR 2019), 2019.

[114] R. Hennequin, A. Khlif, F. Voituret, and M. Moussallam, "Spleeter: a fast and efficient music source separation tool with pre-trained models," J. Open Source Softw., vol. 5, no. 50, 2020.

[115]Q. Zhang and S. Zhu, "Visual interpretability for deep learning: a survey," Frontiers of Inf. Technology \& Electronic Eng., vol. 19, no. 1, pp. 27 39, 2018.

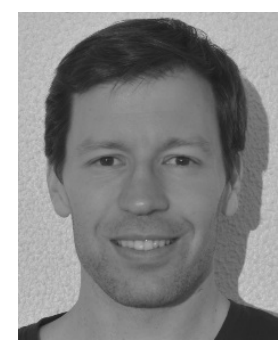

Renato Panda is a PhD from the University of Coimbra, where he also concluded his Master and Bachelor degrees. He currently is an Invited Professor at Polytechnic Institute of Tomar. $\mathrm{He}$ is a member of the Cognitive and Media Systems group at the Center for Informatics and Systems of the University of Coimbra (CISUC). His main research interests are related with Music Emotion Recognition (MER) and Music Information Retrieval (MIR).

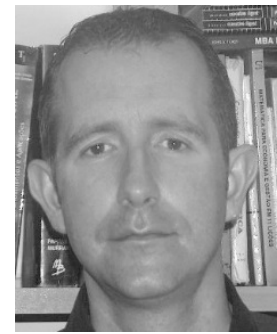

Ricardo Malheiro is a PhD from the University of Coimbra, where he also concluded his Master and Bachelor (Licenciatura - 5 years) degrees, respectively in Informatics Engineering and Mathematics. He is currentlya Professor at Miguel Torga Higher Institute, Coimbra. $\mathrm{He}$ is also a member of the CMS research group at CISUC. His main research interests are in the areas of Natural Language Processing, Detection of Emotions in Music Lyrics and Text and Text/Data Mining.

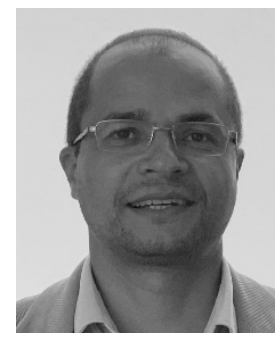

Rui Pedro Paiva is a Professor at the Department of Informatics Engineering of the University of Coimbra, where he concluded his Doctoral, Master and Bachelor degrees in 2007, 1999 and 1996, respectively. $\mathrm{He}$ is also a member of the CMS group at CISUC. His main research interests are in the areas of MIR and Health Informatics. The common research hat is the study of feature engineering, machine learning and signal processing to the analysis of musical and bio signals. 